Víctor Jiménez López, Departamento de Matemáticas, Universidad de Murcia, Campus de Espinardo, Aptdo. de Correos 4021, Murcia 30100, Spain, e-mail: vjimenez@@fcu.um.es

\title{
AN EXPLICIT DESCRIPTION OF ALL SCRAMBLED SETS OF WEAKLY UNIMODAL FUNCTIONS OF TYPE $2^{\infty}$
}

\begin{abstract}
The aim of this paper is to describe in an explicit way all scrambled sets of chaotic weakly unimodal functions of type $2^{\infty}$. For this purpose, we introduce in this setting a new coding method which turns out to be more useful here than the standard one from Milnor and Thurston's kneading theory [12].
\end{abstract}

\section{Introduction}

Since its appearance in 1975 [10], the notion of chaos in the sense of $\mathrm{Li}$ and Yorke has been deeply analyzed, because as is shown in Smítal [17], it gives a very reasonable criterion to decide whether the dynamics of a continuous function $f: I \rightarrow I$ is "complex" or not. Here and in what follows, $I$ will always denote a non-degenerate compact interval. Also, recall that $f^{0}=\mathrm{Id}$, $f^{n}$ is the $n$-th iterate of $f$ and a point $p \in I$ is said to be periodic (of period $r$ ) if there is an $r \geq 1$ such that $f^{r}(p)=p$ and $f^{i}(p) \neq p$ for any $1 \leq i<r$. A periodic point of period 1 is also called a fixed point.

Definition 1 Let $f: I \rightarrow I$ be a continuous function. Suppose that there is an $S \subset I$ with at least two elements such that for any $x, y \in S, x \neq y$, and any periodic point $p$ of $f$ we have

(i) $\limsup _{n \rightarrow \infty}\left|f^{n}(x)-f^{n}(y)\right|>0$,

Mathematical Reviews subject classification: Primary: 58F13. Secondary: 26A18, $54 \mathrm{H} 20$

Received by the editors December 1, 1995

* This work has been partially supported by the DGICYT grant PB91-0575. 
(ii) $\liminf _{n \rightarrow \infty}\left|f^{n}(x)-f^{n}(y)\right|=0$,

(iii) $\limsup _{n \rightarrow \infty}\left|f^{n}(x)-f^{n}(p)\right|>0$.

Then we say that $f$ is chaotic (in the sense of Li and Yorke) and $S$ is called a scrambled set of $f$.

In particular, it is interesting to know whether the size of the scrambled sets of $f$ is "large", since this would imply "observable" chaos. There are several papers showing examples of chaotic functions possessing scrambled sets of positive or even full Lebesgue measure. (See for example Smítal [17], Misiurewicz [14], Bruckner and Hu [3], Jiménez López [7], [8].) However, the natural problem of constructing explicit examples of maximal scrambled sets remained open. Here we say that a scrambled set of $f$ is maximal if it is not properly included in another scrambled set of $f$.

We will need some notation. Throughout the paper, closed intervals are possibly degenerate. Given $A, B \subset I,[A ; B]$ will denote the least interval including $A$ and $B$, while $(A ; B)=[A ; B] \backslash(A \cup B)$. If $A=\{a\}$, we will replace $A$ by $a$ in the above notation and then we will write respectively $[a ; B]$ and $(a ; B)$ (similarly for the case $B=\{b\})$. If $f$ is a function defined on $A$ and $B \subset A,\left.f\right|_{B}$ will denote the restriction of $f$ to $B$.

Definition 2 Let $f: I \rightarrow I$ be a non-constant continuous function, $I=[a ; b]$. We say that $f$ is weakly unimodal if $f(a)=f(b) \in\{a, b\}$ and there is a $c \in(a ; b)$ such that $\left.f\right|_{[a ; c]}$ and $\left.f\right|_{[c ; b]}$ are monotone.

The maximal closed interval $C$ containing $c$ for which $\left.f\right|_{C}$ is constant will be called the turning interval of $f$.

Notice that $\left.f\right|_{[a ; c]}$ need not be strictly monotone (similarly for $\left.f\right|_{[c ; b]}$ ) and likewise the turning interval may be non-degenerate.

Definition 3 Let $f: I \rightarrow I$ be a continuous function. We say that $f$ is of type $2^{\infty}$ if it has periodic points of period $2^{r}$ for every $r \geq 0$ but no other periods.

We will denote by $U(I)$ (resp. $V(I)$ ) the set of weakly unimodal functions $f: I \rightarrow I$ of type $2^{\infty}$ for which all points of the turning interval are absolute maxima (resp. absolute minima). We also define $W(I)=U(I) \cup V(I)$.

It is known that the set $W(I)$ contains some chaotic functions. (See Misiurewicz and Smítal [15].) The aim of this paper is to give an explicit description of all scrambled sets of every chaotic function $f \in W(I)$. Since every scrambled set is a subset of a maximal scrambled set and every subset (containing more than one point) of a scrambled set is also a scrambled set, for this purpose it is sufficient to describe all maximal scrambled sets of $f$. This is 
accomplished in Theorem 1. In order to find such a description we will make use of symbolic techniques, that is, we assign "codes" (sequences of symbols) to points in an appropriate way and study the dynamical properties of the points in terms of the structure of their codes. The standard way of doing this, the kneading theory, is not very useful here. Thus we introduce a more convenient coding method whose details are described in Section 2.

\section{The Coding Method for Weakly Unimodal Functions of Type $2^{\infty}$}

Let $f \in W(I)$ be chaotic. We intend to describe explicitly all its scrambled sets. When trying to solve this problem, we need to be able to identify each point by a specific "name" (or code). Of course we have decimal expansions for this, but they are useless in our setting because they have no relation with the dynamical properties of the points (with respect to $f$ ). Ideally, our coding method should satisfy the following requirements. (a) It must have a "dynamical meaning". (b) Each point must have its corresponding code, or at least each point whose dynamics is significant for our problem. (c) If two points $x, y$ have the same code then their dynamics must be essentially the same; that is, $\lim _{n \rightarrow \infty}\left|f^{n}(x)-f^{n}(y)\right|=0$. (d) For each "reasonable" code there must exist some point having it as its code - this would surely simplify the technical part, both in the statement of results and in the construction of examples.

A standard coding method is provided by Milnor and Thurston's kneading theory [12]. In fact $f$ need not be of type $2^{\infty}$ here. The method consists of assigning an infinite sequence of -1 's, 0 ' $\mathrm{s}$ and 1 's to each point $x$ (its itinerary), with $-1,0$ or 1 being the $n$-th term of the sequence depending on whether $f^{n-1}(x)<C, f^{n-1}(x) \in C$ or $C<f^{n-1}(x)$ ( $C$ the turning interval of $f$ ). Here and in the sequel we use the notation $A<B$ to say that $u<v$ for any $u \in A$ and $v \in B$, where in particular $u<B$ stands for $\{u\}<B$ (similarly for $A<\{v\}$ ). The reader should notice that itineraries are usually defined in the reversed way, that is, the $n$-th term is -1 if $C<f^{n-1}(x)$ and 1 if $f^{n-1}(x)<C$, the reason of our change being to get a consistent notation with that of the rest of the paper.

The dynamical meaning of the sequence is clear, each point has a code and it can be checked that (c) "essentially" holds. However, not every sequence of -1 's, 0 's and 1's need be the itinerary of some point, and this converts kneading theory into rather an uncomfortable tool for our purposes.

So a different approach is needed, taking into account the specific dynamic properties of functions of type $2^{\infty}$. The rest of this section is devoted to describing our coding method and showing that (a)-(d) above holds for it. 
We use the well known idea of renormalization. (See e.g. van Strien [18].) If $f \in W(I)$, then there exists a closed interval $I_{1} \subset I_{0}=I$ such that $f^{2}\left(I_{1}\right) \subset I_{1}$. Moreover, $\left.f^{2}\right|_{I_{1}} \in W\left(I_{1}\right)$. If we let $\Upsilon(f)=\left.f^{2}\right|_{I_{1}}$, inductively define $f_{0}=f$ and $f_{n+1}=\Upsilon\left(f_{n}\right)$ and $I_{n+1}$ is the interval playing the role for $f_{n}$ as $I_{1}$ plays for $f$, then for each point $x \in I$ either it is asymptotically periodic; that is, there is a periodic point $p$ such that $\lim _{i \rightarrow \infty}\left|f^{i}(x)-f^{i}(p)\right|=0$, or for any $n \geq 0$ there is a (minimal) nonnegative integer $l(n)$ such that $x_{n}=f^{l(n)}(x) \in I_{n}$. This allows us to assign to any non-asymptotically periodic point an infinite sequence of integer numbers, which we will denote by $\Pi_{f}(x)$, as follows. If $\alpha_{n}$ is the $n$-th term of the sequence $\Pi_{f}(x)$, this means that $f_{n-1}$ maps $x_{n-1}$ into $I_{n}$ after $\left|\alpha_{n}\right|$ iterations and $f_{n-1}^{\left|\alpha_{n}\right|}\left(x_{n-1}\right)=x_{n}$. If $\alpha_{n}=0$, we have $x_{n-1}=x_{n}$ and then $x_{n-1} \in I_{n}$. Otherwise, $\alpha_{n}$ will be negative or positive depending on whether $x_{n-1}$ lies between $I_{n}$ and the fixed endpoint (for $f_{n-1}$ ) of $I_{n-1}$ or not.

There is still another dynamical interpretation for $\Pi_{f}(x)$ in terms of the renormalization map. Let $\Phi:[0,1] \rightarrow[0,1]$ be defined by $\Phi(x)=\kappa x(1-x)$, where $\kappa \approx 3.5699456 \ldots$ As is well known, $\Phi \in W([0,1])$. (See e.g. Preston [16, p. 189].) Later we will show (Proposition 5) that $f$ is topologically semiconjugate to $\Phi$, that is, there is a monotone onto function $g: I \rightarrow[0,1]$ such that $g \circ f=\Phi \circ g$. Moreover, $\Phi$ and $\Phi_{1}=\Upsilon(\Phi) \in W\left(I^{1}\right)$ are topologically conjugate, which means that the map $h$ with $h \circ \Phi_{1}=\Phi \circ h$ is now a homeomorphism. (See Misiurewicz [13].) Let $J^{1}$ contain $\Phi\left(I^{1}\right)$ and be such that $\Phi\left(J^{1}\right)=I^{1}$. The renormalization map $\nu: I^{1} \cup J^{1} \rightarrow[0,1]$ is defined by $\nu(x)=h(x)$ if $x \in I^{1}$ and $\nu(x)=h(\Phi(x))$ if $x \in J^{1}$. For the set $A$ of points from $I^{1} \cup J^{1}$ whose iterates (by $\nu$ ) always belong to $I^{1} \cup J^{1}$, the map $\nu: A \rightarrow A$ is well defined. In a way which is strongly reminiscent of the kneading theory, we can associate an infinite sequence of 0's and 1's to each $x \in A$, having 0 or 1 as its $n$-th term depending on whether $\nu^{n-1}(x) \in I^{1}$ or $\nu^{n-1}(x) \in J_{1}$. This sequence coincides with $\Pi_{f}(y)$ for any $y \in g^{-1}(\{x\})$.

Let us make the above ideas more precise. Let $f \in W(I), I=[a ; b], f(a)=$ $a$ and $C$ be the turning interval of $f$. Notice that $f([a ; C]) \not \subset[a ; C]$; otherwise $f(I) \subset[a ; C]$ and since $\left.f\right|_{[a ; C]}$ is increasing, $f$ could only have periodic points of period 1. Then there exists a (unique) fixed point $p$ of $f$ such that $p \in$ $(C ; b)$. (See Figure 1) Now take $q \in[C ; p]$ with $f^{2}(q)=q$ and $f^{2}(x) \neq x$ for any $x \in(C ; q)$. Observe that $q \notin C$. If not, for any $x \in[a ; q]$ either $f^{k}(x) \in[q ; f(q)]$ for some $k$ or $\left(f^{n}(x)\right)_{n=0}^{\infty}$ would be monotone. This, together with $f([f(q) ; b])=[a ; q]$ and the fact that $\left.f\right|_{[q ; f(q)]}:[q ; f(q)] \rightarrow[q ; f(q)]$ is decreasing (and then it can only have periodic points of periods 1 and 2 ), leads to a contradiction. A similar reasoning shows that

$$
f^{2}(x)<q \quad \text { for any } x \in(C ; q)
$$

since otherwise $f^{2}(x) \in[x ; f(x)]$ for any $x \in[C ; q]$. Now take $u \in(a ; C)$ such that $f(u)=f(q)$ and $f(x) \neq f(q)$ for any $x \in(u ; C)$. Since $f$ is of type $2^{\infty}$, 


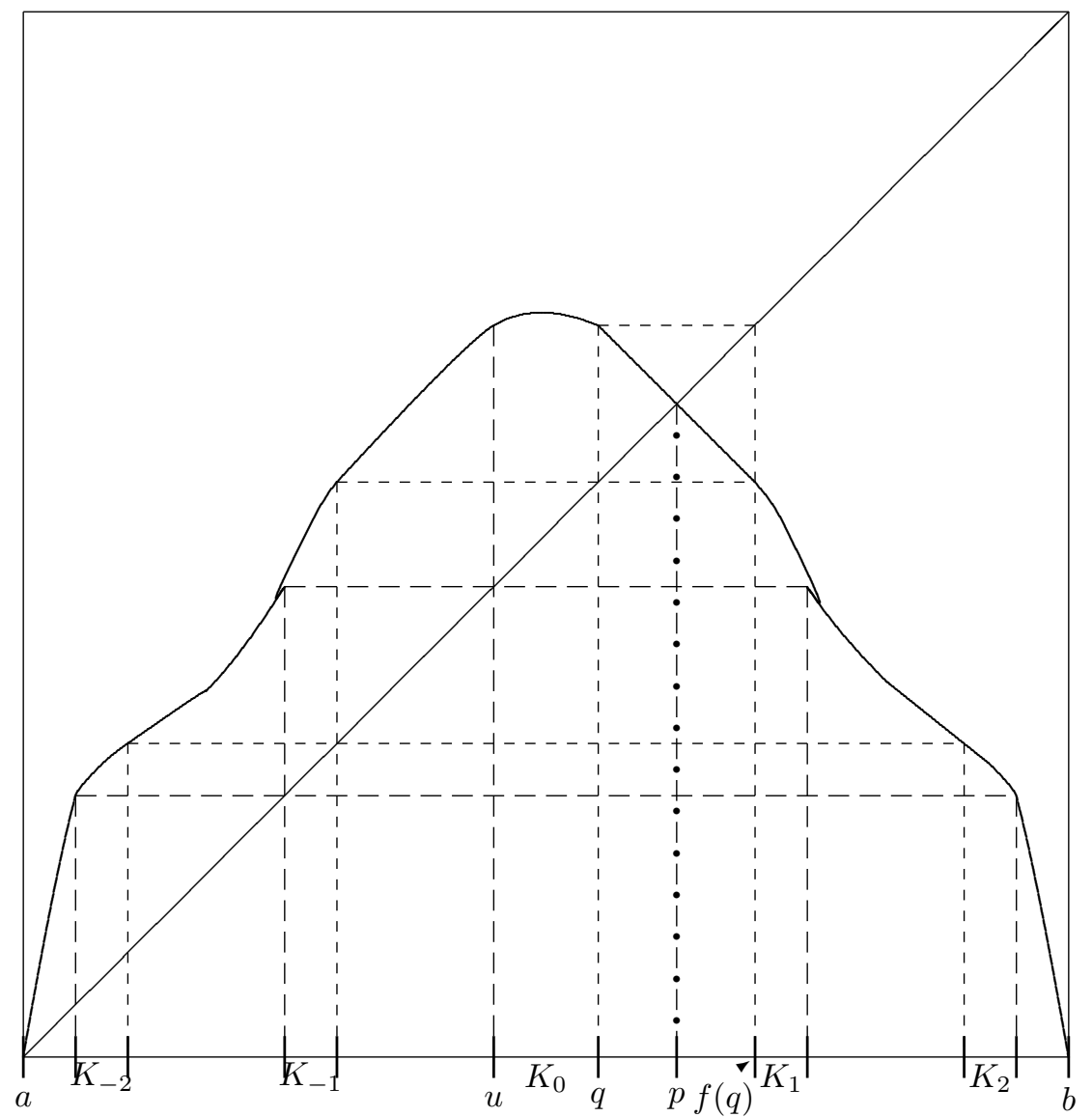

Figure 1: The graph of a function $f \in U(I)$; here $K_{m}$ stands for $K_{m}(f)$ for any $m$. 
$f^{2}(C) \subset(u ; q)$. (See Šarkovskii [19] or Block [2].) Thus $f^{2}([u ; q]) \subset[u ; q]$. The interval $[u ; q]$ is denoted by $I_{1}$.

So the function $\Upsilon(f)=\left.f^{2}\right|_{I_{1}}: I_{1} \rightarrow I_{1}$ is well defined. By arguments similar to those in the above paragraph, it is easy to show that $\Upsilon(f) \in W\left(I_{1}\right)$. More precisely, $\Upsilon(f) \in V\left(I_{1}\right)$ if $f \in U(I)$ and $\Upsilon(f) \in U\left(I_{1}\right)$ if $f \in V(I)$.

Before continuing, we give some necessary notations and definitions.

For any set $A, A^{\infty}$ and $A^{n}$ denote as usual the set of infinite sequences and finite sequences of length $n$ of points from $A$ and Card $A$ will be its cardinality. If $\alpha$ is such a sequence, we denote its $k$-th term by $\alpha_{k}$. If $\alpha$ is infinite or its length is larger than $n$, we set $\left.\alpha\right|_{n}=\alpha_{1}, \alpha_{2}, \ldots, \alpha_{n}$. The shift map $\sigma: A^{\infty} \rightarrow$ $A^{\infty}$ is defined by $\sigma(\alpha)=\alpha_{2}, \alpha_{3}, \alpha_{4}, \ldots$.

Let $\mathbb{Z}$ denote the set of integers. If $\alpha \in \mathbb{Z}^{n}$, we put $\tau(\alpha)=\sum_{i=1}^{n}\left|\alpha_{i}\right| 2^{i-1}$, and $\alpha, l$ will denote the sequence $\alpha_{1}, \alpha_{2}, \ldots, \alpha_{n}, l$ for any integer $l$. The sequences $\mathbf{0}, \mathbf{1} \in \mathbb{Z}^{\infty}$ are defined by $\mathbf{0}_{i}=0$ and $\mathbf{1}_{i}=1$ for any $i$.

We order the sets $\mathbb{Z}^{\infty}, \mathbb{Z}^{n}$ as follows. If $\alpha, \beta \in \mathbb{Z}^{\infty}$ or $\alpha, \beta \in \mathbb{Z}^{n}, \alpha \neq \beta$ and $k$ is the first integer such that $\alpha_{k} \neq \beta_{k}$, we say that $\alpha<\beta$ if either $k+\operatorname{Card}\left\{1 \leq i<k: \alpha_{i}>0\right\}$ is odd and $\alpha_{k}<\beta_{k}$ or $k+\operatorname{Card}\{1 \leq i<$ $\left.k: \alpha_{i}>0\right\}$ is even and $\beta_{k}<\alpha_{k}$. For instance, let $\alpha=2,0,-1,0,5, \ldots$, $\beta=2,0,-1,0,-3, \ldots$ In this case $k=5$ and Card $\left\{1 \leq i<5: \alpha_{i}>0\right\}=1$. Since 6 is even and $\beta_{5}<\alpha_{5}$, we have $\alpha<\beta$. Observe that in the case $n=1$ (when we identify $\mathbb{Z}^{1}$ and $\mathbb{Z}$ ), $<$ is the usual order in $\mathbb{Z}$.

Finally let us introduce some specific notation for $f$. Recall that the sequence $\left(f_{n}\right)_{n=0}^{\infty}$ was defined inductively by $f_{0}=f$ and $f_{n+1}=\Upsilon\left(f_{n}\right)$. For any $n \geq 1$ and $\alpha \in \mathbb{Z}^{n}$ define the set $K_{\alpha}(f)$ as follows. Put $K_{0}(f)=(u ; q)$ and inductively define $K_{-m}(f)$ and $K_{m}(f)$ by $K_{-m}(f)=f^{-1}\left(K_{-m+1}(f)\right) \cap(a ; C)$ and $K_{m}(f)=f^{-1}\left(K_{-m+1}(f)\right) \cap(C ; b)$ for any $m \geq 1$. Now assume that $K_{\alpha}(h)$ has been already defined for any $\alpha \in \bigcup_{i=1}^{n} \mathbb{Z}^{i}$, any compact interval $K$ and any $\tilde{f} \in W(K)$ and put

$$
K_{\alpha}(f)=\left\{x \in K_{\left.\alpha\right|_{n}}(f): f^{\tau\left(\left.\alpha\right|_{n}\right)}(x) \in K_{\alpha_{n+1}}\left(f_{n}\right)\right\}
$$

for any $\alpha \in \mathbb{Z}^{n+1}$. Finally, define $K_{\alpha}(f)=\bigcap_{n=1}^{\infty} K_{\left.\alpha\right|_{n}}(f)$ for any $\alpha \in \mathbb{Z}^{\infty}$ and put $K(f)=\bigcup_{\alpha \in \mathbb{Z}_{\infty}} K_{\alpha}(f)$.

Let us explain the connection of the above sets with our problem. First we emphasize that $\mathrm{Cl}\left(K_{0}\left(f_{n-1}\right)\right)=I_{n}$ for any $n \geq 1$. (Here $\mathrm{Cl} A$ denotes the closure of a set $A$.) Note that $\mathrm{Cl}\left(K_{1}(f)\right)=g^{-1}\left(J^{1}\right)$ with the notation we introduced before defining the renormalization map. Take an arbitrary $\alpha \in \mathbb{Z}^{\infty}$ and let $x \in K_{\alpha}(f)$. Then $x \in K_{\alpha_{1}}(f)$ and $\left|\alpha_{1}\right|$ is the necessary number of iterations of $f$ to map $x$ into $I_{1}$. Further, $\alpha_{1}$ is negative or positive depending on whether $x$ lies between $I_{1}$ and $a$ or $I_{1}$ and $b$. Put $x_{1}=f^{\left|\alpha_{1}\right|}(x)$. Then $x_{1} \in K_{\alpha_{2}}\left(f_{1}\right)$ and $\left|\alpha_{2}\right|$ is the necessary number of iterations of $f_{1}$ to map $x_{1}$ into $I_{2}$. The sign of $\alpha_{2}$ tells us whether $x_{1}$ lies between $I_{2}$ and the fixed 
endpoint of $f_{1}$ or not. In general, if we define $x_{n}=f_{n-1}^{\left|\alpha_{n}\right|}\left(x_{n-1}\right)$ and repeat the process, we find that it takes $\left|\alpha_{n+1}\right|$ iterations of $f_{n}$ to map $x_{n}$ into $I_{n+1}$ and the sign of $\alpha_{n}$ describes the relative position of $x_{n}$ with respect to $I_{n+1}$. Moreover, $\tau\left(\left.\alpha\right|_{n}\right)$ is the first number such that $f^{\tau\left(\left.\alpha\right|_{n}\right)}(x) \in I_{n}$. (This is the number we denoted at the beginning of the section by $l(n)$.) In other words, $\alpha$ is the code $\Pi_{f}(x)$ we were looking for.

There are still some problems to solve. Let us recall them. (A) $\Pi_{f}$, seen as a map from $K(f)$ to $\mathbb{Z}^{\infty}$, must be well defined, or equivalently, the sets $K_{\alpha}(f), \alpha \in \mathbb{Z}^{\infty}$, must be pairwise disjoint. (B) $K(f)$ must coincide with the set of non-asymptotically periodic points of $f$. (C) If $x, y \in K_{\alpha}(f)$ for some $\alpha \in \mathbb{Z}^{\infty}$, then we must have $\lim _{n \rightarrow \infty}\left|f^{n}(x)-f^{n}(y)\right|=0$. (D) $\Pi_{f}$ must be surjective; that is, $K_{\alpha}(f) \neq \emptyset$ for any $\alpha \in \mathbb{Z}^{\infty}$. Notice that (B), (C) and (D) correspond to the requirements (b), (c) and (d) from the beginning of this section. (With respect to (b) we remark that a scrambled set cannot contain any asymptotically periodic points and so these points are negligible for our purposes.)

The following proposition summarizes some useful properties of the sets $K_{\alpha}(f), \alpha \in \mathbb{Z}^{n}$, and in particular allows us to solve (A) and (D). Indeed, if $\alpha \neq \beta$, then $\left.\alpha\right|_{k} \neq\left.\beta\right|_{k}$ for some $k$, and by (iii) below $K_{\left.\alpha\right|_{k}}(f) \cap K_{\left.\beta\right|_{k}}(f)=\emptyset$ which also implies $K_{\alpha}(f) \cap K_{\beta}(f)=\emptyset$. This solves (A). From (i) and (iii), $\mathrm{Cl}\left(K_{\left.\alpha\right|_{n+1}}(f)\right) \subset K_{\left.\alpha\right|_{n}}(f)$ for any $n$ and $\alpha \in \mathbb{Z}^{\infty}$ and then (i) implies that $K_{\alpha}(f)$ is a closed interval, in particular a nonempty set. This proves (D).

Proposition 1 Let $f \in W(I)$ and $C$ be its turning interval. Then for any $n \geq 1$ and any $\alpha \in \mathbb{Z}^{n}$, the following properties hold.

(i) $K_{\alpha}(f)$ is an open interval.

(ii) $K_{\mathbf{0} \mid n}(f)=K_{0}\left(f_{n-1}\right)$. In particular, $C \subset K_{\mathbf{0} \mid{ }_{n}}(f)$.

(iii) Let $\beta \in \mathbb{Z}^{n}$. If $f \in U(I)$ (resp. $f \in V(I)$ ) and $\alpha<\beta$, then $K_{\alpha}(f)<$ $K_{\beta}(f)\left(\right.$ resp. $\left.K_{\beta}(f)<K_{\alpha}(f)\right)$.

(iv) Suppose $\alpha \neq\left.\mathbf{0}\right|_{n}$ and let $j$ be the first integer such that $\alpha_{j} \neq 0$. Define $\beta \in \mathbb{Z}^{n}$ by $\beta_{i}=1$ for any $i<j, \beta_{j}=1-\left|\alpha_{j}\right|$ and $\beta_{i}=\alpha_{i}$ for any $i>j$. Then $\left.f\left(K_{\alpha}(f)\right)=K_{\beta}(f)\right)$; in particular, $f^{\tau(\alpha)}\left(K_{\alpha}(f)\right)=K_{\left.\mathbf{0}\right|_{n}}(f)$ (because $\tau\left(\left.\beta\right|_{n}\right)=\tau\left(\left.\alpha\right|_{n}\right)-1$ ). Additionally, $f\left(K_{\left.\mathbf{0}\right|_{n}}(f)\right) \subset K_{\left.\mathbf{1}\right|_{n}}(f)$.

Proof. We use induction on $n$. Indeed we will also prove the following auxiliary property.

(v) If Card $\left\{1 \leq i \leq n: \alpha_{i}>0\right\}$ is even (resp. odd), then $\left.f^{\tau(\alpha)}\right|_{K_{\alpha}(f)}$ is increasing (resp. decreasing). 
Properties (i)-(v) clearly hold for the case $n=1$ (use (1) to check $\left.f\left(K_{0}(f)\right) \subset K_{1}(f)\right)$. Now, supposed that Proposition 1 holds for every $n \leq k$. We will prove it in the case $n=k+1$.

To begin with, (ii) is trivial, while (i) is an immediate consequence of the particular case in (iv) for $n=k$. To prove (iii), we can assume $\left.\alpha\right|_{k}=\left.\beta\right|_{k}$ since otherwise it follows from the induction hypothesis. For example assume that $f \in U(I)$ and $k$ is odd. (The other possibilities are analogous.) Since $f_{k} \in V\left(\mathrm{Cl}\left(K_{0}\left(f_{k-1}\right)\right)\right), m<l$ implies $K_{l}\left(f_{k}\right)<K_{m}\left(f_{k}\right)$ for any $m, l \in \mathbb{Z}$. Using (v) for $n=k$, (iii) follows.

To prove (iv), we will distinguish two cases. If $\left.\alpha\right|_{k} \neq\left.\mathbf{0}\right|_{k}$, we get $f\left(K_{\left.\alpha\right|_{k}}(f)\right)$ $=K_{\left.\beta\right|_{k}}(f)$ by (iv) for the case $n=k$. Since $\tau\left(\left.\beta\right|_{k}\right)=\tau\left(\left.\alpha\right|_{k}\right)-1$ and $\alpha_{k+1}=$ $\beta_{k+1}$, we clearly get $f\left(K_{\alpha}(f)\right)=K_{\beta}(f)$.

Now assume $\alpha=\left.\mathbf{0}\right|_{k}, l$. As in the paragraph above,

$$
f^{2^{k}-1}\left(K_{\left.\mathbf{1}\right|_{k}}(f)\right)=K_{\left.\mathbf{0}\right|_{k}}(f), \quad f^{2^{k}-1}\left(K_{\left.\mathbf{1}\right|_{k}, m}(f)\right)=K_{\left.\mathbf{0}\right|_{k}, m}(f)
$$

for any $m \in \mathbb{Z}$. Further, notice that

$$
f^{2^{k}-1}\left(K_{\left.\mathbf{1}\right|_{k}}(f) \backslash K_{\left.\mathbf{1}\right|_{k}, m}(f)\right)=K_{\left.\mathbf{0}\right|_{k}}(f) \backslash K_{\left.\mathbf{0}\right|_{k}, m}(f) .
$$

By (iv) for $n=k$, we also get

$$
f\left(K_{\left.\mathbf{0}\right|_{k}}(f)\right) \subset K_{\left.\mathbf{1}\right|_{k}}(f) .
$$

On the other hand, $K_{\alpha}(f)=K_{l}\left(f_{k}\right)$ and so

$$
f^{2^{k}}\left(K_{\alpha}(f)\right)=K_{\left.\mathbf{0}\right|_{k}, 1-|l|}(f)
$$

if $l \neq 0$ and

$$
f^{2^{k}}\left(K_{\left.\mathbf{0}\right|_{k+1}}(f)\right) \subset K_{\left.\mathbf{0}\right|_{k}, 1}(f) .
$$

If $l \neq 0,(2)-(5)$ imply $f\left(K_{\alpha}(f)\right) \subset K_{\beta}(f)$. Further, $f\left(K_{\alpha}(f)\right)=K_{\beta}(f)$ since otherwise some endpoint of $K_{l}\left(f_{k}\right)$ would be mapped by $f_{k}$ into $K_{1-|l|}\left(f_{k}\right)$, a contradiction. Similarly, $f\left(K_{\left.\mathbf{0}\right|_{k+1}}(f)\right) \subset K_{\left.\mathbf{1}\right|_{k+1}}(f)$.

Finally, (v) follows trivially from the induction hypothesis if $\alpha_{k+1}=0$. For example assume that Card $\left\{1 \leq i \leq k: \alpha_{i}>0\right\}$ is even and $\alpha_{k+1}>0$ (We omit the other cases.), when Card $\left\{1 \leq i \leq k+1: \alpha_{i}>0\right\}$ is odd. Then $\left.f_{k}^{\alpha_{k+1}}\right|_{K_{\alpha_{k+1}}\left(f_{k}\right)}$ is clearly decreasing. Since $\left.f^{\tau\left(\left.\alpha\right|_{k}\right)}\right|_{K_{\alpha}(f)}$ is increasing by (v) for $n=k,\left.f^{\tau(\alpha)}\right|_{K_{\alpha}(f)}=\left.\left(f^{2^{k} \cdot \alpha_{k+1}} \circ f^{\tau\left(\left.\alpha\right|_{k}\right)}\right)\right|_{K_{\alpha}(f)}$ is decreasing.

Sometimes, if $\alpha \in\{0,1\}^{n}$, then we will denote $K_{\alpha}(f)$ by $K_{j}^{n}(f)$, where $j=2^{n}-\tau(\alpha)$, and also $K_{2^{n}}^{n}(f)=K_{\mathbf{0} \mid}(f)$ by $K_{0}^{n}(f)$. Then for any $n$, Proposition 1 (iii) and (iv) imply respectively

$$
\mathrm{Cl}\left(K_{i}^{n+1}(f) \cup K_{i+2^{n}}^{n+1}(f)\right) \subset K_{i}^{n}(f) \quad \text { for any } 0 \leq i<2^{n},
$$




$$
f\left(K_{0}^{n}(f)\right) \subset K_{1}^{n}(f) \text { and } f\left(K_{i}^{n}(f)\right)=K_{i+1}^{n}(f) \text { for any } 1 \leq i<2^{n} .
$$

Also, it will be useful to reinterpret Proposition 1 in terms of the sets $K_{\alpha}(f)$ but now with $\alpha \in \mathbb{Z}^{\infty}$. For instance, (ii) gives

$$
C \subset K_{\mathbf{0}}(f)
$$

$C$ the turning interval of $f$. From (iii) we get an easy characterization of the order $<$ in $\mathbb{Z}^{\infty}: \alpha<\beta$ if and only if $K_{\alpha}(f)<K_{\beta}(f)$ (in the case $f \in U(I)$ ), and $\alpha<\beta$ if and only if $K_{\beta}(f)<K_{\alpha}(f)$ (in the case $f \in V(I)$ ). If $\alpha \in \mathbb{Z}^{\infty}, \alpha \neq \mathbf{0}$, $j$ is the first integer such that $\alpha_{j} \neq 0$ and we define $\beta \in \mathbb{Z}^{\infty}$ by $\beta_{i}=1$ for any $i<j, \beta_{j}=1-\left|\alpha_{j}\right|$ and $\beta_{i}=\alpha_{i}$ for any $i>j$, then

$$
f\left(K_{\alpha}(f)\right)=K_{\beta}(f) .
$$

To prove it, use Proposition 1 (iv) to get $f\left(K_{\left.\alpha\right|_{n}}(f)\right)=K_{\left.\beta\right|_{n}}(f)$ for any $n \geq j$, while as in (3), $f\left(K_{\left.\alpha\right|_{n}}(f) \backslash K_{\left.\alpha\right|_{n+1}}(f)\right)=K_{\left.\beta\right|_{n}}(f) \backslash K_{\left.\beta\right|_{n+1}}(f)$ for any $n \geq j$. Similarly,

$$
f\left(K_{\mathbf{0}}(f)\right) \subset K_{\mathbf{1}}(f) .
$$

The following proposition solves (B).

Proposition 2 Let $f \in W(I)$ and $x \in I$. Then $x \in K(f)$ if and only if it is a non-asymptotically periodic point.

Proof. Define $a, b$ and $q$ as at the beginning of this section. Then we clearly have that for any $x \in\left(K_{m}(f) ; K_{m+1}(f)\right), m \leq 0$ (resp. $\left.m>0\right), f^{|m|}(x) \in$ $[q ; f(q)]$ (resp. $f^{m+1}(x) \in[q ; f(q)]$ ). Further, if $[a ; c]$ and $[d ; b]$ are maximal with the property $[a ; c] \cap \bigcup_{m=-\infty}^{\infty} K_{m}(f)=\emptyset=[d ; b] \cap \bigcup_{m=-\infty}^{\infty} K_{m}(f)$, then $c$ is a fixed point of $f$ and $f([a ; c])=[a ; c], f([d ; b])=[a ; c]$. Hence, every $x \in I \backslash \bigcup_{m=-\infty}^{\infty} K_{m}(f)$ is asymptotically periodic. Reasoning similarly for every $f_{n}$ (Also use Proposition 1 (ii) and (iv).), we get that all points of $I \backslash K(f)$ are asymptotically periodic.

Conversely, take $x \in K(f)$ and assume that $\lim _{n \rightarrow \infty}\left|f^{n}(x)-f^{n}(p)\right|=0$ for some periodic point $p$ of period $2^{r}$. By (7), there exists a $k$ large enough so that $f^{k}(x) \in \cup_{i=0}^{2^{r+2}-1} K_{i}^{r+2}(f)$. Since by (6) the sets $K_{j}^{r+2}(f) \cup K_{j+2^{r+1}}^{r+2}(f)$, $0 \leq j<2^{r+1}$, have disjoint closures, we arrive at a contradiction.

Recall that if $f: I \rightarrow I$ is a continuous function, we say that a nondegenerate subinterval $J$ of $I$ is a wandering interval of $f$ if $f^{n}(J) \cap f^{m}(J)=\emptyset$ for any $n>m \geq 0$ and it does not contain any asymptotically periodic points. In particular, the lengths of $f^{n}(J)$ tend to 0 when $n \rightarrow \infty$ and so $\lim _{n \rightarrow \infty}\left|f^{n}(x)-f^{n}(y)\right|=0$ for any $x, y \in J$. Then the following proposition solves $(\mathrm{C})$. 
Proposition 3 Let $f \in W(I)$. If for an $\alpha \in \mathbb{Z}^{\infty}$ the interval $K_{\alpha}(f)$ is non-degenerate, then it is a wandering interval of $f$. Conversely, if $J$ is a wandering interval of $f$, then there is an $\alpha \in \mathbb{Z}^{\infty}$ such that $J \subset K_{\alpha}(f)$.

Proof. From (9) and Proposition 1(iii), we easily get $f^{n}\left(K_{\alpha}(f)\right) \cap f^{m}\left(K_{\alpha}(f)\right)$ $=\emptyset$ for any $n>m \geq 0$ and any $\alpha \in \mathbb{Z}^{\infty}$. From this and Proposition 2, the proposition follows.

Here e would like to draw the reader's attention to the following problem. As we will show in the next section, the chaoticity of a map $f \in W(I)$ depends essentially on the existence of non-degenerate intervals $K_{\alpha}(f)$ for some special $\alpha \in \mathbb{Z}^{\infty}$. Therefore it is important to know whether given some set $\mathcal{A} \subset \mathbb{Z}^{\infty}$ there exists a map $f \in W(I)$ with the property that $K_{\alpha}(f)$ is non-degenerate if and only if $\alpha \in \mathcal{A}$.

Of course $\mathcal{A}$ cannot be arbitrary. For instance it must be countable. Further, if $\alpha$ and $\beta$ are such that $f\left(K_{\alpha}(f)\right)=K_{\beta}(f)$ (compare with (9)), $\beta \in \mathcal{A}$ implies $\alpha \in \mathcal{A}$. By the way, notice that $f\left(K_{\mathbf{0}}(f)\right) \subset K_{\mathbf{1}}(f)$ by (10) but $f\left(K_{\mathbf{0}}(f)\right)=K_{\mathbf{1}}(f)$ does not necessarily hold, so $\mathbf{1} \in A$ need not imply $\mathbf{0} \in A$. This suggests the following definition. Let $\alpha, \beta \in \mathbb{Z}^{\infty}$ and fix some $f \in W(I)$. We say that $\alpha \preceq \beta$ if there is a $k \geq 0$ such that $f^{k}\left(K_{\alpha}(f)\right)=K_{\beta}(f)$ and $f^{n}\left(K_{\alpha}(f)\right) \neq K_{\mathbf{0}}(f)$ for any $0 \leq n<k$. Notice that $\preceq$ does not depend on the choice of $f$ and has no relation with the order $<$ defined above. Also, we say that a set $\mathcal{A} \subset \mathbb{Z}^{\infty}$ is closed for $\preceq$ if $\alpha \in \mathcal{A}$ and $\beta \preceq \alpha$ imply $\beta \in \mathcal{A}$. Observe that if $K_{\alpha}(f)$ is non-degenerate and $\beta \preceq \alpha$, then $\bar{K}_{\beta}(f)$ is non-degenerate as well.

Thus we have that for any $f \in W(I)$ the set of sequences $\alpha$ for which $K_{\alpha}(f)$ is non-degenerate is countable and closed for $\preceq$. It turns out that the converse also holds.

Proposition 4 Let $\mathcal{A} \subset \mathbb{Z}^{\infty}$ be closed for $\preceq$ and countable. Then there is a function $f \in W([0,1])$ such that, for any $\alpha \in \mathbb{Z}^{\infty}, \alpha \in \mathcal{A}$ if and only if $K_{\alpha}(f)$ is non-degenerate.

Proof. We will use the technique of blowing-up orbits. (See e.g. Harrison [6].) Namely, let $\Phi$ be the parabola of type $2^{\infty}$ defined at the beginning of this section. It is well known that $\Phi$ has no wandering intervals. (See Martens, de Melo and van Strien [11] or Misiurewicz [13].) According to Proposition 3, all intervals $K_{\alpha}(\Phi), \alpha \in \mathbb{Z}^{\infty}$, are degenerate. Since $\mathcal{A}$ is countable, it is easy to construct an increasing onto function $g:[0,1] \rightarrow[0,1]$ such that $g^{-1}(\{u\})$ is non-degenerate if and only if $\{u\}=K_{\alpha}(\Phi)$ for some $\alpha \in \mathcal{A}$. Now let us define $f:[0,1] \rightarrow[0,1]$ in the following way. Let $\alpha, \beta \in \mathbb{Z}^{\infty}$ be such that $\Phi\left(K_{\alpha}(\Phi)\right)=K_{\beta}(\Phi), \alpha<\mathbf{0}($ resp. $\mathbf{0}<\alpha)$. Then we define $f$ on $g^{-1}\left(K_{\alpha}(\Phi)\right)$ in such a way that $f\left(g^{-1}\left(K_{\alpha}(\Phi)\right)\right)=g^{-1}\left(K_{\beta}(\Phi)\right)$ and $\left.f\right|_{g^{-1}\left(K_{\alpha}(\Phi)\right)}$ is increasing 
(resp. decreasing). Since $\mathcal{A}$ is closed for $\preceq$, if $g^{-1}\left(K_{\beta}(\Phi)\right)$ is non-degenerate, then so is $g^{-1}\left(K_{\alpha}(\Phi)\right)$, and the above construction makes sense. Further, write $g^{-1}\left(K_{\mathbf{1}}(\Phi)\right)=[a ; b], a \leq b$, and define $f(x)=a$ for any $x \in g^{-1}\left(K_{\mathbf{0}}(\Phi)\right)$. Finally, if $u \in[0,1] \backslash K(\Phi), \Phi(u)=v$ and $g(x)=u, g(y)=v$, then we define $f(x)=y$ (notice that from Proposition $2, v \in[0,1] \backslash K(\Phi)$ and then there is no ambiguity about $y$ ). By (8) and (10) (for $\Phi), f$ is continuous and weakly unimodal. Moreover, $g \circ f=\Phi \circ g$ and then $f$ is clearly of type $2^{\infty}$. (Use (9) and (10).)

In the proof of the above proposition, for a given $g$ we constructed an appropriate function $f$ topologically semiconjugate to $\Phi$ via $g$. Conversely, any function $f \in W(I)$ is topologically semiconjugate to $\Phi$. This result was proved earlier by Misiurewicz and Smítal in [15], who used it in the same paper to show that any function $f \in W(I)$ having a non-degenerate turning interval is chaotic (cf. also [5]). (By the way, notice that this last result follows as a particular case from Theorem 1(i).) It turns out that Misiurewicz and Smítal's proof of the semiconjugacy property contains a mistake. Namely, for every weakly unimodal function $f: I \rightarrow I$ they consider the equivalence relation $\sim$ defined in $I$ as follows: $x \sim y$ if there is a $k$ such that $f^{k}$ is constant on the closed interval containing $x$ and $y$. They state that every equivalence class is a closed interval and use this property later. But it is not true in general. To see this, as in the proof of Proposition 4, it suffices to construct an $f \in U([0,1])$ for which there is an increasing onto function $g:[0,1] \rightarrow[0,1]$ with $g \circ f=\Phi \circ g$ and such that $g([0,1 / 3])=0, g([2 / 3,1])=1$ and $\left.g\right|_{[1 / 3,2 / 3]}$ is one-to-one. Notice that $f$ can be defined arbitrarily on $[0,1 / 3]$, except that $\left.f\right|_{[0,1 / 3]}$ must be increasing and $f(0)=0, f(1 / 3)=1 / 3$. In particular we can assume $f(x)=2 x$ for any $x \in[0,1 / 6]$ and $f(x)=1 / 3$ for any $x \in[1 / 6,1 / 3]$. Since $f((1 / 3,2 / 3)) \subset(1 / 3,2 / 3)$, it is obvious that the class containing $1 / 3$ is $(0,1 / 3]$.

For completeness we give here a proof of the above result, whose essential ideas were suggested to the author by one of the referees.

Proposition 5 Any function $f \in W(I)$ is topologically semiconjugate to $\Phi$.

Proof. We can assume $f \in U(I)$. For any $x \in I$, let us define the sequence $\left(\theta_{f}^{n}(x)\right)_{n=1}^{\infty}$ similarly to the itinerary of $x$ in the kneading theory, the only difference being that $K_{\mathbf{0}}(f)$ now plays the role of the turning interval $C$ of $f$. More precisely, $\theta_{n}(x)$ equals $-1,0$ or 1 depending on whether $f^{n-1}(x)<$ $K_{\mathbf{0}}(f), f^{n-1}(x) \in K_{\mathbf{0}}(f)$ or $K_{\mathbf{0}}(f)<f^{n-1}(x)$. We denote by $K_{f}(x)$ the set of points from $I$ for which its corresponding sequence coincides with that of $x$.

Clearly, $K(x)$ is an interval and

$$
f\left(K_{f}(x)\right) \subset K_{f}(f(x))
$$


by (9) and (10). We claim that

$$
K_{f}(x) \text { is closed for any } x \text {. }
$$

In fact, it is easy to check that $K_{f}(x)=K_{\Pi_{f}(x)}(f)$ if $x$ is a non-asymptotically periodic point and then (12) holds for these points. In general, observe that if $K_{f}(x)$ is not closed, then there is a $k$ such that $f^{k}\left(K_{f}(x)\right) \cap K_{\mathbf{0}}(f)=\emptyset$ but $\mathrm{Cl}\left(f^{k}\left(K_{f}(x)\right)\right) \cap K_{\mathbf{0}}(f) \neq \emptyset$. By $(11)$, this implies that $K_{f}\left(f^{k}(x)\right)$ includes infinitely many $K_{\alpha}(f)$ 's, which is impossible.

By (11), we can construct an increasing onto function $g: I \rightarrow[0,1]$ such that $g\left(K_{f}(x)\right)$ is a single point for any $x$ and $g\left(K_{f}(x)\right) \neq g\left(K_{f}(y)\right)$ if $x \neq y$. By (12), there is a continuous function $h:[0,1] \rightarrow[0,1]$ with $g \circ f=h \circ g$. It is easy to show that $h \in U([0,1])$. (Recall $f \in U(I)$ and use (6) and (7).) Clearly, each $K_{h}(u)$ is degenerate. Then $\left(\theta_{h}^{n}(u)\right)_{n=1}^{\infty}$ is the standard itinerary of $u$ and different points from $[0,1]$ have different itineraries (with respect to $h$ ). It is well known that $\Phi$ has the same property. (See [13]). Further, from the structure of maps from $U([0,1])$ follows that the set of itineraries of points from $[0,1]$ are the same both with respect to $h$ and $\Phi$. Then $h$ and $\Phi$ are topologically conjugate. (Use e.g. Lemmas II.1.2 and II.1.3 from [4].)

\section{Maximal Scrambled Sets for Weakly Unimodal Func- tions of Type $2^{\infty}$}

In order to simplify the notation, we will write $\Pi, K_{\alpha}, K_{j}^{n}$ and $K$ instead of $\Pi_{f}, K_{\alpha}(f), K_{j}^{n}(f)$ and $K(f)$ throughout the rest of the paper (except in Lemmas 2 and 4$)$. Since in each case $f$ will be clearly stated, this should not lead to confusion. Proposition 1 and (6)-(10) will be repeatedly used without further references.

In order to get an explicit description of all maximal scrambled sets of a function $f \in W(I)$ we need, according to Proposition 2, to characterize explicitly (in terms of their codes) the points $x, y \in K$ such that $\lim _{n \rightarrow \infty} \mid f^{n}(x)-$ $f^{n}(y) \mid=0$ and and those for which $\liminf _{n \rightarrow \infty}\left|f^{n}(x)-f^{n}(y)\right|=0$. (More briefly, we will write respectively in these cases $x \sim_{f}^{s} y$ and $x \sim_{f}^{i} y-$ or simply $x \sim^{s} y$ and $x \sim^{i} y$, as above not referring specifically to $f$.) In fact it is obvious that $\sim^{s}$ is an equivalence relation in $K$ and Proposition 6 below describes explicitly $\sim^{i}$ and shows that it is an equivalence relation as well. We call every equivalence class for $\sim^{s}$ (resp. $\sim^{i}$ ) a $\sim_{f^{-}}^{s}$ class $\left(\right.$ resp. $\sim_{f^{-}}^{i}$ class $)$ or simply a $\sim^{s}$-class (resp. $\sim^{i}$-class). Thus each maximal scrambled set of $f$ is characterized by the property of being included in some $\sim^{i}$-class $\mathrm{E}$ and containing exactly one representative from every $\sim^{s}$-class included in $E$.

For any given $k, f^{n}(x), f^{n}(y) \in \bigcup_{j=0}^{2^{k}-1} K_{j}^{k}$ if $n$ is large enough. Then Proposition 6 says in other words that $x \sim^{s} y$ if and only if there are no 
$n$ and $k$ such that $f^{n}(x), f^{n}(y)$ belong to different $K_{j}^{k}, K_{l}^{k}$. We could also consider Proposition 6 in the light of the semiconjugacy $g$ between $f$ and $\Phi$ (cf. Proposition 5). Then $x \sim^{i} y$ if and only if the sequences $\left(\Phi^{2^{n}}(g(x))\right)_{n}$ and $\left(\Phi^{2^{n}}(g(y))\right)_{n}$ have the same limit.

Proposition 6 Let $f \in W(I), x, y \in K, \alpha=\Pi(x), \beta=\Pi(y)$. Then $x \sim^{i} y$ if and only if $\tau\left(\left.\alpha\right|_{i}\right) \equiv \tau\left(\left.\beta\right|_{i}\right)\left(\bmod 2^{i}\right)$ for any $i$.

Proof. Let us prove the "only if" part. Assume the contrary. Then there is a $k$ such that

$$
\tau\left(\left.\alpha\right|_{k}\right) \not \equiv \tau\left(\left.\beta\right|_{k}\right) \quad\left(\bmod 2^{k}\right) .
$$

Put $n_{1}=\tau\left(\left.\alpha\right|_{k}\right), n_{2}=\tau\left(\left.\beta\right|_{k}\right)$. Let us say $n_{1}>n_{2}$. From (13) we get $f^{n_{1}}(x) \in K_{0}^{k}, f^{n_{1}}(y) \in K_{l}^{k}$ for some $0<l<2^{k}$, and so $f^{n_{1}+m 2^{k}}(x) \in$ $K_{0}^{k}, f^{n_{1}+m 2^{k}}(y) \in K_{l}^{k}$ for any $m$. Take $j$ large enough so that $n_{1}+j 2^{k} \geq$ $\max \left\{\tau\left(\left.\alpha\right|_{k+1}\right), \tau\left(\left.\beta\right|_{k+1}\right)\right\}$. Then $f^{n_{1}+m 2^{k}}(x) \in K_{0}^{k+1} \cup K_{2^{k}}^{k+1}, f^{n_{1}+m 2^{k}}(y) \in$ $K_{l}^{k+1} \cup K_{l+2^{k}}^{k+1}$ for any $m \geq j$. Since these sets have disjoint closures, we get a contradiction.

Now we show the "if" part. Fix $\epsilon>0$ and choose $K_{l}^{k}=(a, b)$ with $b-a<\epsilon$. As above, put $n_{1}=\tau\left(\left.\alpha\right|_{k}\right), n_{2}=\tau\left(\left.\beta\right|_{k}\right), n_{1} \geq n_{2}$. Since $n_{1}=n_{2}+j 2^{k}$ for some $j \geq 0$, we get $f^{n_{1}+l+m 2^{k}}(x), f^{n_{1}+l+m 2^{k}}(y) \in K_{l}^{k}$ for any $m$, which finishes the proof.

Thus it remains only to find an explicit characterization of $\sim^{s}$. To begin with, assume that $K_{\gamma}$ is non-degenerate for some $\gamma \in \mathbb{Z}^{\infty}$. If $x \sim^{s} y$, there must exists a $k$ large enough such that $K_{\gamma} \not \subset\left(f^{n}(x), f^{n}(y)\right)$ for any $n \geq k$, or equivalently, there must exist an $l$ large enough such that $K_{\gamma} \cap\left[f^{n}(x), f^{n}(y)\right]=$ $\emptyset$ for any $n \geq l$ (due to Proposition 3 ). We will describe this situation by saying that $x \sim_{\gamma, f}^{s} y$, or simply $x \sim_{\gamma}^{s} y$. On the contrary we will write as usual $x \chi_{\gamma}^{s} y$. Obviously, $\sim_{\gamma}^{s}$ defines an equivalence relation in $K$.

So it seems reasonable to begin by getting information about $\sim_{\gamma}^{s}$ for any $\gamma \in \mathbb{Z}^{\infty}$. (Notice that the definition of $\sim_{\gamma}^{s}$ makes sense both if $K_{\gamma}$ is degenerate and if not.) For many $\gamma$ 's such information is easy to obtain, but useless. $\left(x \sim^{i} y\right.$ (in particular $\left.x \sim^{s} y\right)$ implies automatically $x \sim_{\gamma}^{s} y$ for any $x, y \in K$.) This is clearly the case if $\gamma \notin\{0,1\}^{\infty}$. The same thing happens if $\gamma \in\{0,1\}^{\infty}$ and $\sigma^{k}(\gamma)=\mathbf{1}$ for some $k$; that is, $\gamma_{i}=1$ for any $i>k$. To prove it we can for example assume $f \in U(I)$. Suppose $x \sim^{i} y$ but $x \not_{\gamma}^{s} y$ for some $x, y \in$ $K$. Since $\gamma \in\{0,1\}^{\infty}$, there is an $l \geq 0$ such that $\left.f^{l}\right|_{K_{\left.1\right|_{k}}}$ is monotone and $f^{l}\left(K_{\left.\mathbf{1}\right|_{k}}\right)=K_{\left.\gamma\right|_{k}}$. Choose $n$ large enough such that $f^{n-l}(x), f^{n-l}(y) \in K_{\left.\mathbf{1}\right|_{k}}$, $f^{n}(x), f^{n}(y) \in K_{\left.\gamma\right|_{k}}$ and $K_{\gamma} \subset\left(f^{n}(x) ; f^{n}(y)\right)$. Since $f^{l}\left(K_{\mathbf{1}}\right)=K_{\gamma}$, either $K_{1}<f^{n-l}(x)$ or $K_{1}<f^{n-l}(y)$, which is impossible because $f(I) \subset[a, b]$, 
where $a$ and $b$ are respectively the left endpoint of $I$ and the right endpoint of $K_{1}$.

This leads us to the set

$$
\mathcal{S}=\left\{\gamma \in\{0,1\}^{\infty}: \sigma^{i}(\gamma) \neq \mathbf{1} \text { for any } i\right\} .
$$

One could wonder whether $\gamma, \theta \in \mathcal{S}$ can induce the same equivalence relation, that is, $x \sim_{\gamma}^{s} y$ if and only if $x \sim_{\theta}^{s} y$ for any $x, y \in K$. Note that this property does not depend on the choice of $f$. In this situation we say that $\gamma \sim_{e} \theta$ and call every equivalence class for $\sim_{e}$ a $\sim_{e}$-class. Notice that all the equivalence relations we had introduced earlier $\left(\sim^{i}, \sim^{s}, \sim_{\gamma}^{s}\right)$ are defined in $K$, but $\sim_{e}$ is defined in $\mathcal{S}$.

We know that if $x \sim^{s} y$, then $x \sim_{\gamma}^{s} y$ for any $\gamma \in \mathcal{S}$ for which $K_{\gamma}$ is non-degenerate. This suggests the following. Define $\gamma \in \mathcal{S}$ to be an essential sequence of $f$ if $K_{\gamma}$ is non-degenerate and call a $\sim_{e}$-class containing some essential sequence of $f$ an essential class of $f$. With this notation, a necessary condition for $x \sim^{s} y$ is that, fixed a set $\mathcal{E}$ containing exactly one representative from every essential class of $f, x \sim_{\gamma}^{s} y$ for every $\gamma \in \mathcal{E}$. The following proposition shows that this condition is also sufficient (provided $x \sim^{i} y$ ) and characterizes $\sim^{s}$. As we will see in Lemma 3 below, that $x \sim_{\gamma}^{s} y$ for some $\gamma \in \mathcal{E}$ implies $x \sim^{i} y$, but this condition is necessary in the case $\mathcal{E}=\emptyset$.

Proposition 7 Let $f \in W(I), x, y \in K$ and $\mathcal{E} \subset \mathcal{S}$ be a set containing exactly one representative from every essential class of $f$. Then $x \sim^{s} y$ if and only if $x \sim^{i} y$ and $x \sim_{\gamma}^{s}$ y for any $\gamma \in \mathcal{E}$.

Proof. It remains only to prove the "if part". Assume that there are some $x, y \in K$ such that $x \sim^{i} y$ but for which $x \sim^{s} y$ does not hold. Then there are an $\epsilon>0$ and a strictly increasing sequence $(n(j))_{j=1}^{\infty}$ such that $\left|f^{n(j)}(x)-f^{n(j)}(y)\right|>\epsilon$ for any $j$. Take the intervals $\left[f^{n(j)}(x) ; f^{n(j)}(y)\right]$ and consider the sequence $\left(c_{j}\right)_{j=1}^{\infty}$ of their midpoints. Let $c$ be an accumulation point of $\left(c_{j}\right)_{j=1}^{\infty}$. There is no loss of generality in assuming that $\left|c-c_{j}\right|<\epsilon / 4$ for any $j$. Thus

$$
[c-\epsilon / 4, c+\epsilon / 4] \subset\left[f^{n(j)}(x) ; f^{n(j)}(y)\right] \quad \text { for any } j .
$$

On the other hand, from $x \sim^{i} y$ and Proposition 6 we can assume that there are a strictly increasing sequence $(m(j))_{j=1}^{\infty}$ and a $\gamma[j] \in\{0,1\}^{m(j)}$ such that for every $j\left[f^{n(j)}(x) ; f^{n(j)}(y)\right] \subset K_{\gamma[j]}$. Then $[c-\epsilon / 4, c+\epsilon / 4] \subset K_{\gamma[j]}$, from which we get that $K_{\gamma[j+1]} \cap K_{\gamma[j]} \neq \emptyset$ for any $j$. Therefore there is a $\gamma \in\{0,1\}^{\infty}$ such that $\left.\gamma\right|_{m(j)}=\gamma[j]$ for any $j$ and with the property

$$
[c-\epsilon / 4, c+\epsilon / 4] \subset K_{\gamma} .
$$


So $K_{\gamma}$ is non-degenerate. From (14) and (15) we get $K_{\gamma} \cap\left[f^{n(j)}(x) ; f^{n(j)}(y)\right] \neq$ $\emptyset$ for any $j$. Thus $x \chi_{\gamma}^{s} y$. But we have shown at the beginning of this section that if $\theta \notin \mathcal{S}$, then $x \sim^{i} y$ implies $x \sim_{\theta}^{S} y$. Hence, $\gamma \in \mathcal{S}$. This finishes the proof.

Although Proposition 7 characterizes $\sim^{s}$, it is not sufficient for our purposes because we are aiming for an explicit description. This means that we must explicitly characterize $\sim_{\gamma}^{s}$ for any $\gamma \in \mathcal{S}$ and $\sim_{e}$. This is accomplished in Propositions 8 and 9.

Fix $\gamma \in \mathcal{S}$. Let us explain the main points of the characterization of $\sim_{\gamma}^{s}$. First of all, we take advantage of the fact that $\sigma^{i}(\gamma) \neq \mathbf{1}$ for any $i$ to define the strictly increasing sequence $\left(o_{\gamma}(j)\right)_{j=1}^{\infty}$ (or simply $(o(j))_{j=1}^{\infty}$ if there is no confusion about $\gamma$ ) of the indexes $i$ with the property $\gamma_{i}=0$. For instance, suppose

$$
\gamma=1,1,1,0,0,0,0,1,1,1,1,0,1,1,0,1,0,1,0,1,0,1,0, \ldots
$$

Then $o(1)=4, o(2)=5, o(3)=6, o(4)=7, o(5)=12, o(6)=15$ and $o(j)=2 j+3$ for any $j \geq 7$. The next step is to use this sequence to "cut" each $\alpha \in \mathbb{Z}^{\infty}$ into the blocks $\alpha_{\gamma}^{j}=\alpha_{o(j-1)+1}, \alpha_{o(j-1)+2}, \ldots, \alpha_{o(j)}$ (or simply $\left.\alpha^{j}\right)$; here we mean $o(0)=0$. These blocks occupy the same places as those of $\gamma$ maximal with the property that the last term is 0 and all the other terms are 1's. So in the above case we get $\gamma^{1}=1,1,1,0, \gamma^{2}=0, \gamma^{3}=0, \gamma^{4}=0$, $\gamma^{5}=1,1,1,1,0, \gamma^{6}=1,1,0$, and $\gamma^{j}=1,0$ for any $j \geq 7$, and if for example

$$
\alpha=2,2,4,0,3,0,2,0,1,0,1,0,2,5,1,-1,0,-1,0,-1,0,-1,0, \ldots,
$$

then $\alpha^{1}=2,2,4,0, \alpha^{2}=3, \alpha^{3}=0, \alpha^{4}=2, \alpha^{5}=0,1,0,1,0, \alpha^{6}=2,5,1$ and $\alpha^{j}=-1,0$ for any $j \geq 7$.

The above decomposition in blocks turns out to be very useful to describe $\sim_{\gamma}^{s}$. Let $x, y \in K, \Pi(x)=\alpha, \Pi(y)=\beta$ and suppose $x \sim_{\gamma}^{s} y$. We will show that there must exists a $k$ large enough such that $\tau\left(\left.\alpha\right|_{o(k)}\right)=\tau\left(\left.\beta\right|_{o(k)}\right)$ and $\tau\left(\alpha^{j}\right)=\tau\left(\beta^{j}\right)$ for any $j>k$. For instance suppose

$$
\begin{aligned}
& \alpha=2,5,1,5,1,5,1,5,1, \ldots, \\
& \beta=2,3,2,3,2,3,2,3,2, \ldots, \\
& \gamma=1,0,1,0,1,0,1,0,1, \ldots
\end{aligned}
$$

Then $\alpha^{1}=2,5, \beta^{1}=2,3$ and $\alpha^{j}=1,5, \beta^{j}=2,3$ for any $j \geq 2$. Since $\tau(1,5)=11$ and $\tau(2,3)=10, x \mathcal{\psi}_{\gamma}^{s} y$. Note that $x \sim^{i} y$ and that $\tau\left(\left.\alpha\right|_{i}\right)=$ $\tau\left(\left.\beta\right|_{i}\right)$ for any odd number $i$. In fact we prove the above result under the additional hypothesis that both sequences $\left(\tau\left(\left.\alpha\right|_{i}\right)\right)_{i=1}^{\infty},\left(\tau\left(\left.\beta\right|_{i}\right)\right)_{i=1}^{\infty}$ are unbounded, or equivalently, $\sigma^{i}(\alpha) \neq \mathbf{0} \neq \sigma^{i}(\beta)$ for any $i$. Note that this condition cannot 
be dropped. For instance, define

$$
\begin{aligned}
\alpha & =\mathbf{0}, \\
\beta & =2,1,1,1,1,1,1, \ldots, \\
\eta & =0,1,1,1,1,1,1, \ldots .
\end{aligned}
$$

Since $f^{2}\left(K_{\alpha}\right) \subset K_{\eta}=f^{2}\left(K_{\beta}\right)$, by Proposition 3 we get $x \sim_{\theta}^{s} y$ for any $\theta \in \mathbb{Z}^{\infty}$ and any $x \in K_{\alpha}, y \in K_{\beta}$.

Fortunately, there is a simple way to deal with this problem, which is indeed suggested by the above example. For any $\alpha \in \mathbb{Z}^{\infty}$, define $\mathrm{n}(\alpha) \in \mathbb{Z}^{\infty}$ by

$$
\mathrm{n}(\alpha)=\left\{\begin{array}{l}
2+\sup _{i} \tau\left(\left.\alpha\right|_{i}\right), 1,1,1,1,1,1, \ldots \text { if }\left(\tau\left(\left.\alpha\right|_{i}\right)\right)_{i=1}^{\infty} \text { is bounded } \\
\mathrm{n}(\alpha)=\alpha \text { otherwise. }
\end{array}\right.
$$

If $x \in K_{\alpha}, x^{\prime} \in K_{\mathrm{n}(\alpha)}$, we get as before that there are some appropriate $l$ and $\eta$ such that $f^{l}(x), f^{l}\left(x^{\prime}\right) \in K_{\eta}$ (namely $l=2+\sup _{i} \tau\left(\left.\alpha\right|_{i}\right)$ and $\eta=$ $0,1,1,1,1,1,1, \ldots$ as above) and so $x \sim_{\gamma}^{s} y$ if and only if $x^{\prime} \sim_{\gamma}^{s} y$. In other words, we can study $\sim_{\gamma}^{s}$ in terms of the properties not of the sequences $\Pi(x)$ 's but of $\mathrm{n}(\Pi(x))$ 's. So our previous result can be restated in the following way. Let $x, y \in K, \mathrm{n}(\Pi(x))=\theta, \mathrm{n}(\Pi(y))=\vartheta$ and suppose $x \sim_{\gamma}^{s} y$. Then there must exists a $k$ large enough such that $\tau\left(\left.\theta\right|_{o(k)}\right)=\tau\left(\left.\vartheta\right|_{o(k)}\right)$ and $\tau\left(\theta^{j}\right)=\tau\left(\vartheta^{j}\right)$ for any $j>k$.

However, the above necessary condition is not sufficient. To go further for any $\alpha \in \mathbb{Z}^{\infty}$ we must introduce a sequence $\delta_{\gamma}^{\alpha} \in\{-1,0,1\}^{\infty}$ (or simply $\delta^{\alpha}$ ) as follows. Most of the terms of the sequence $\delta^{\alpha}$ will be 0 's; namely we put $\delta_{i}^{\alpha}=0$ if $i \neq o(j)$ for any $j \geq 1$. (Recall that $(o(j))_{j=1}^{\infty}$ is the sequence of indexes for which the corresponding term of $\gamma$ is 0 .) Now suppose that $i=o(j)$ for some $j \geq 1$ and define $\delta_{i}^{\alpha}$. To do this we first take into account the "size" (in terms of $\tau$ ) of the block $\alpha^{j}$. Then if $\tau\left(\alpha^{j}\right)<2^{i-o(j-1)-1}$ (that is, if $\tau\left(\alpha^{j}\right)<2^{l-1}$, $l$ the length of $\alpha^{j}$ ), we define again $\delta_{i}^{\alpha}=0$. It remains only to consider the case $\tau\left(\alpha^{j}\right) \geq 2^{i-o(j-1)-1}$. (We have this situation if for example $\alpha_{i} \neq 0$.) It can happen that $\alpha^{j}$ is the last "large" block; that is, $\tau\left(\alpha^{m}\right)<2^{o(m)-o(m-1)-1}$ for any $m>j$. Then we again put $\delta_{i}^{\alpha}=0$. Otherwise let $m>j$ be minimal such that $\tau\left(\alpha^{m}\right) \geq 2^{o(m)-o(m-1)-1}$. Note that $\tau\left(\gamma^{m}\right)=2^{o(m)-o(m-1)-1}-1$ and in particular $\alpha^{m} \neq \gamma^{m}$. Now the main role is played (with one exception) by the relative position of the finite sequences $\alpha_{i+1}, \alpha_{i+2}, \ldots, \alpha_{o(m)}$ and $\gamma_{i+1}, \gamma_{i+2}, \ldots, \gamma_{o(m)}$. These sequences are constructed by simply putting together respectively the blocks $\alpha^{j+1}, \alpha^{j+2}, \ldots, \alpha^{m}$ and $\gamma^{j+1}, \gamma^{j+2}, \ldots, \gamma^{m}$. Since $\alpha^{m} \neq \gamma^{m}$, the sequences are different. Now we define $\delta_{i}^{\alpha}=1$ if $\alpha_{i}=0$ or $\gamma_{i+1}, \gamma_{i+2}, \ldots, \gamma_{o(m)}<\alpha_{i+1}, \alpha_{i+2}, \ldots, \alpha_{o(m)}$ and $\delta_{i}^{\alpha}=-1$ if $\alpha_{i} \neq 0$ and $\alpha_{i+1}, \alpha_{i+2}, \ldots, \alpha_{o(m)}<\gamma_{i+1}, \gamma_{i+2}, \ldots, \gamma_{o(m)}$. This finishes the definition of $\delta^{\alpha}$. 
As an example, let us return to the sequences

$$
\begin{aligned}
& \gamma=1,1,1,0,0,0,0,1,1,1,1,0,1,1,0,1,0,1,0,1,0,1,0, \ldots \\
& \alpha=2,2,4,0,3,0,2,0,1,0,1,0,2,5,1,-1,0,-1,0,-1,0,-1,0, \ldots
\end{aligned}
$$

considered above. First notice that $\tau\left(\alpha^{1}\right)=\tau(2,2,4,0)=22 \geq 8=$ $2^{o(1)-o(0)-1}$. Further, $\tau\left(\alpha^{2}\right)=\tau(3)=3 \geq 1=2^{o(2)-o(1)-1}$. So since $\alpha_{o(1)}=0$ we get $\delta_{1}^{\alpha}=0, \delta_{2}^{\alpha}=0, \delta_{3}^{\alpha}=0, \delta_{4}^{\alpha}=\delta_{o(1)}^{\alpha}=1$. Next, $\tau\left(\alpha^{3}\right)=\tau(0)=0$, $\tau\left(\alpha^{4}\right)=\tau(2)=2 \geq 1=2^{o(4)-o(3)-1}$. Since $\alpha_{o(2)} \neq 0$ and $0,2<0,0$, we get $\delta_{5}^{\alpha}=\delta_{o(2)}^{\alpha}=-1, \delta_{6}^{\alpha}=\delta_{o(3)}^{\alpha}=0$. Similarly, $\tau\left(\alpha^{5}\right)=\tau(0,1,0,1,0)=10<$ $16=2^{o(5)-o(4)-1}, \tau\left(\alpha^{6}\right)=\tau(2,5,1)=16 \geq 4=2^{o(6)-o(5)-1}$. Again $\alpha_{o(4)} \neq 0$ and $0,1,0,1,0,2,5,1<1,1,1,1,0,1,1,0$ so $\delta_{7}^{\alpha}=\delta_{o(4)}^{\alpha}=-1, \delta_{8}^{\alpha}=0, \delta_{9}^{\alpha}=0$, $\delta_{10}^{\alpha}=0, \delta_{11}^{\alpha}=0, \delta_{12}^{\alpha}=\delta_{o(5)}^{\alpha}=0, \delta_{13}^{\alpha}=0$ and $\delta_{14}^{\alpha}=0$. Finally, notice that $\tau\left(\alpha^{m}\right)<2^{o(m)-o(m-1)-1}$ for any $m>6$. Thus $\delta_{15}^{\alpha}=\delta_{o(6)}^{\alpha}=0$ and also $\delta_{i}^{\alpha}=0$ for any $i \geq 16$. Briefly,

$$
\delta^{\alpha}=0,0,0,1,-1,0,-1,0,0,0,0,0,0,0,0,0, \ldots
$$

With the help of the sequences $\delta^{\alpha}$ we can finish our explicit characterization of $\sim_{\gamma}^{s}: x \sim_{\gamma}^{s} y$ if and only if the necessary condition described above holds and $\delta_{i}^{\theta}=\delta_{i}^{\vartheta}$ for any $i$ large enough. Summarizing we have the following.

Proposition 8 Let $f \in W(I), \gamma \in \mathcal{S}, x, y \in K, \alpha=\Pi(x), \beta=\Pi(y)$. Also put $\theta=\mathrm{n}(\alpha), \vartheta=\mathrm{n}(\beta)$. Then $x \sim_{\gamma}^{s} y$ if and only if there is an $m$ such that $\tau\left(\left.\theta\right|_{o(m)}\right)=\tau\left(\left.\vartheta\right|_{o(m)}\right), \tau\left(\theta^{j}\right)=\tau\left(\vartheta^{j}\right)$ for any $j>m$ and $\delta_{i}^{\theta}=\delta_{i}^{\vartheta}$ for any $i>o(m)$.

Before proving Proposition 8 let us still make some additional observations. Recall that $\sim_{\gamma}^{s}$ defines an equivalence relation in $K$. Then, informally speaking, Proposition 8 says that the "size" of every equivalence class for $\sim_{\gamma}^{s}$ is directly related with the "abundance" of 0 's in the sequence $\gamma$. As the extremal cases, recall that if $\sigma^{k}(\gamma)=\mathbf{1}$ for some $k, x \sim^{i} y$ implies $x \sim_{\gamma}^{s} y$. On the other hand, if $\sigma^{k}(\gamma)=\mathbf{0}$ for some $k$ and $x \sim_{\gamma}^{s} y$, we get from Proposition 8 that there is $l$ such that $\tau\left(\left.\theta\right|_{l}\right)=\tau\left(\left.\vartheta\right|_{l}\right)$ and $\sigma^{l}(\theta)=\sigma^{l}(\vartheta)$; that is, there are $\eta \in \mathbb{Z}^{\infty}$ and $n$ such that $f^{n}(x), f^{n}(y) \in K_{\eta}$.

Observe that the description becomes substantially more complicated in the cases when the sequences $\alpha$ and $\beta$ contains some 0's and 1's. If $\alpha, \beta \in$ $(\mathbb{Z} \backslash\{0,1\})^{\infty}$, we can restate Proposition 8 as follows. $x \sim_{\gamma}^{s} y$ if and only if there is an $m$ such that $\tau\left(\left.\alpha\right|_{o(m)}\right)=\tau\left(\left.\beta\right|_{o(m)}\right)$ and $\tau\left(\alpha^{j}\right)=\tau\left(\beta^{j}\right)$ and both $\alpha_{o(j)+1}, \beta_{o(j)+1}$ have the same sign for any $j>m$. In this case, it is also possible to reinterpret $\sim_{\gamma}^{s}$ without making use of codes. (Although of course they are being implicitly used because we assume $\alpha, \beta \in(\mathbb{Z} \backslash\{0,1\})^{\infty}$.) 
Returning to the notation at the beginning of Section 2 , let $l_{x}(n), l_{y}(n)$ be the minimal numbers such that $f^{l_{x}(n)}(x), f^{l_{y}(n)}(y) \in I_{n}$. Then $x \sim_{\gamma}^{s} y$ if and only if $l_{x}(o(j))=l_{y}(o(j))$ and the turning interval of $f$ does not lies between $f^{l_{x}(o(j))}(x)$ and $f^{l_{y}(o(j))}(y)$ for any $j$ large enough.

Finally, a few words about the proof of Proposition 8. It follows from a sequence of lemmas (Lemmas 1-5). Lemmas 1 and 2 are of a technical nature, the first one stating an almost trivial but useful fact and the second one aiming for a better handle of the considerably complicated notation. Lemma 3 states that if $x, y \in K$ and $x \sim_{\gamma}^{s} y$, then $x \sim^{i} y$. Since $\tau\left(\left.\alpha\right|_{k}\right) \equiv \tau\left(\left.\beta\right|_{k}\right)\left(\bmod 2^{k}\right)$ obviously implies $\tau\left(\left.\alpha\right|_{i}\right) \equiv \tau\left(\left.\beta\right|_{i}\right)\left(\bmod 2^{i}\right)$ for any $i<k$, this is in fact a particular case of Lemma 4 . However, we have preferred to state it in an independent way both because it is necessary for proving Lemma 4 and to emphasize that this is not necessarily the case for a general $\gamma \in \mathbb{Z}^{\infty}$. For instance if $\gamma_{1}>1$ and $\Pi(x)=\alpha$, then $f^{n}(x)<K_{\gamma}$ for any $n \geq \alpha_{1}$. Therefore, $x \sim_{\gamma}^{s} y$ for any $x, y \in K$. Finally, the proposition is proved in Lemmas 4 and 5 .

Recall that for every $f \in W(I)$ the sequence $\left(f_{n}\right)_{n=0}^{\infty}$ was defined at the beginning of Section 2 .

Lemma 1 Let $k \geq 0$ and $J$ be a subinterval of $I$ such that $\left.f^{k}\right|_{J}$ is monotone. Let $u, v \in J$ and $\eta, \rho \in \mathbb{Z}^{\infty}$ be such that $K_{\eta} \subset J$ and $f^{k}\left(K_{\eta}\right)=K_{\rho}$. If $K_{\rho} \cap\left[f^{k}(u) ; f^{k}(v)\right] \neq \emptyset$, then $K_{\eta} \cap[u ; v] \neq \emptyset$.

Proof. Suppose not. For example suppose that $u \leq v, K_{\eta}<[u ; v]$ and $f^{k}$ is increasing (the other cases are similar). Then we must have $f^{k}(u) \in K_{\rho}$, which implies that $f^{k}(z) \in K_{\rho}$ for any $K_{\eta}<z<u$. Since $K_{\eta}=\bigcap_{n=1}^{\infty} K_{\left.\eta\right|_{n}}$, there must exist an $l$ large enough so that $w<u$ for the right endpoint $w$ of $K_{\left.\eta\right|_{l}}$. This contradicts Propositions 2 and 3.

Lemma 2 Let $r \geq 0$ and $u, v \in K_{0}^{k}(f)$ for some $k$. Then

$$
K_{\gamma}(f) \cap\left[f^{n}(u) ; f^{n}(v)\right]=\emptyset \quad \text { for any } 0<n \leq r 2^{k}
$$

if and only if

$$
K_{\sigma^{k}(\gamma)}\left(f_{k}\right) \cap\left[f_{k}^{j}(u) ; f_{k}^{j}(v)\right]=\emptyset \quad \text { for any } 0<j \leq r .
$$

Proof. Define $\eta \in \mathbb{Z}^{\infty}$ by $\left.\eta\right|_{k}=\left.\mathbf{0}\right|_{k}$ and $\sigma^{k}(\eta)=\sigma^{k}(\gamma)$. Let us prove the "only if" part. Suppose that there is some $0<l \leq r$ such that $K_{\sigma^{k}(\gamma)}\left(f_{k}\right) \cap$ $\left[f_{k}^{l}(u) ; f_{k}^{l}(v)\right] \neq \emptyset$. Since $K_{\sigma^{k}(\gamma)}\left(f_{k}\right)=K_{\eta}(f)$ and $f_{k}=\left.f^{2^{k}}\right|_{\mathrm{Cl}\left(K_{\left.\mathbf{0}\right|_{k}}(f)\right)}$, we get

$$
K_{\eta}(f) \cap\left[f^{l 2^{k}}(u) ; f^{l 2^{k}}(v)\right] \neq \emptyset
$$


Say $K_{\left.\gamma\right|_{k}}(f)=K_{s}^{k}(f), 0<s \leq 2^{k}$. (Recall that $K_{0}^{k}=K_{2^{k}}^{k}$.) So $f^{s+(l-1) 2^{k}}(u)$, $f^{s+(l-1) 2^{k}}(v) \in K_{\left.\gamma\right|_{k}}(f)$. Further, $f^{2^{k}-s}\left(K_{\gamma}(f)\right)=K_{\eta}(f)$. Since $\left.f^{2^{k}-s}\right|_{K_{\left.\gamma\right|_{k}}(f)}$ is monotone, (16) and Lemma 1 imply

$$
K_{\gamma}(f) \cap\left[f^{s+(l-1) 2^{k}}(u) ; f^{s+(l-1) 2^{k}}(v)\right] \neq \emptyset .
$$

Let us prove the "if" part. Suppose that there is a $0<j \leq r 2^{k}$ such that $K_{\gamma}(f) \cap\left[f^{j}(u) ; f^{j}(v)\right] \neq \emptyset$. We have $f^{j}(u), f^{j}(v) \in K_{s}^{k}(f)$ for some s. Since $K_{\gamma}(f) \cap K_{s}^{k}(f) \neq \emptyset, K_{\gamma}(f) \subset K_{s}^{k}(f)$ and so $K_{s}^{k}(f)=K_{\left.\gamma\right|_{k}}(f)$. Then, if $(l-1) 2^{k}<j \leq l 2^{k}$, we get $f^{l 2^{k}-j}\left(K_{\gamma}(f)\right)=K_{\eta}(f)$. So $K_{\eta}(f) \cap$ $\left[f^{l 2^{k}}(u) ; f^{l 2^{k}}(v)\right] \neq \emptyset$; that is, $K_{\sigma^{k}(\gamma)}\left(f_{k}\right) \cap\left[f_{k}^{l}(u) ; f_{k}^{l}(v)\right] \neq \emptyset$.

Lemma 3 If $x \sim_{\gamma}^{s} y$, then $x \sim^{i} y$.

Proof. Suppose not. Then, according to Proposition 6, there must exist some intervals $K_{i_{1}}^{k} \neq K_{i_{2}}^{k}$ and an $s$ large enough so that $K_{\gamma} \subset K_{i_{1}}^{k}$ and $f^{s+n 2^{k}}(x) \in K_{i_{1}}^{k}, f^{s+n 2^{k}}(y) \in K_{i_{2}}^{k}$ for any $n$. Moreover, for some $l>k$ large enough there exist $K_{j_{1}}^{l}<K_{j_{2}}^{l}<K_{j_{3}}^{l}$ such that $K_{j_{r}}^{l} \subset K_{i_{1}}^{k}$ for any $r \in\{1,2,3\}$ and $K_{\gamma} \subset K_{j_{2}}^{l}$. (Use $\gamma \in \mathcal{S}$.) Also, we can choose numbers $n_{1}, n_{2}$ as large as necessary so that $f^{s+n_{1} 2^{k}}(x) \in K_{j_{1}}^{l}$ and $f^{s+n_{2} 2^{k}}(x) \in K_{j_{3}}^{l}$. This contradicts $x \sim_{\gamma}^{s} y$.

Lemma 4 Suppose that $x \sim_{\gamma}^{s} y$ and $\alpha=\theta, \beta=\vartheta$. Then there is a $k$ such that $\tau\left(\left.\alpha\right|_{o(k)}\right)=\tau\left(\left.\beta\right|_{o(k)}\right)$ and $\tau\left(\alpha^{j}\right)=\tau\left(\beta^{j}\right)$ for any $j>k$.

Proof. Take an $l$ such that $K_{\gamma}(f) \cap\left[f^{n}(x) ; f^{n}(y)\right]=\emptyset$ for any $n>l$ and choose $k_{0}$ large enough so that $\tau\left(\left.\alpha\right|_{o\left(k_{0}\right)}\right)>l, \tau\left(\left.\beta\right|_{o\left(k_{0}\right)}\right)>l$. (This is possible because $\alpha=\theta, \beta=\vartheta$.) For example, assume that there is a $t>k_{0}$ such that $r=\tau\left(\left.\alpha\right|_{o(t)}\right)<\tau\left(\left.\beta\right|_{o(t)}\right)$. By modifying $t$ if necessary it may be assumed that $\tau\left(\left.\beta\right|_{o\left(k_{0}\right)}\right)<r$. Put $u=f^{r}(x), v=f^{r}(y)$. Then $u \in K_{0}^{i}(f)$ for any $i \leq o(t)$. Similarly $v \in K_{j}^{o\left(k_{0}\right)}(f)$ for some $j$. Then Lemma 3 and Proposition 6 imply $v \in K_{0}^{o\left(k_{0}\right)}(f)$. Now take an $s \geq o\left(k_{0}\right)$ maximal with respect to the property $v \in K_{0}^{s}(f)$. Since $\tau\left(\left.\alpha\right|_{o(t)}\right)<\tau\left(\left.\beta\right|_{o(t)}\right)$, we get $s<o(t)$. Then $u \in K_{\left.\mathbf{0}\right|_{o(t)-s}}\left(f_{s}\right)$, while $v \in K_{w}\left(f_{s}\right)$ for some $w \in \mathbb{Z} \backslash\{0\}$. Moreover, $|w|>1$ from $x \sim^{i} y$. For example assume that $f_{s} \in U\left(\mathrm{Cl}\left(K_{\left.\mathbf{0}\right|_{s}}(f)\right)\right)$. Then clearly $f_{s}(v)<K_{\sigma^{s}(\gamma)}\left(f_{s}\right)$. Recall that $f_{s}\left(K_{\left.\mathbf{0}\right|_{o(t)-s}}\left(f_{s}\right)\right) \subset K_{\left.\mathbf{1}\right|_{o(t)-s}}\left(f_{s}\right)$. Since $\gamma_{o(t)}=0, K_{\sigma^{s}(\gamma)}\left(f_{s}\right)<f_{s}(u)$. This contradicts Lemma 2 .

In the following lemma we mean $o(0)=0$ and $\tau\left(\left.\alpha\right|_{0}\right)=\tau\left(\left.\beta\right|_{0}\right)=0$.

Lemma 5 Suppose that $\tau\left(\left.\alpha\right|_{o(k-1)}\right)=\tau\left(\left.\beta\right|_{o(k-1)}\right), \tau\left(\left.\alpha\right|_{o(k)}\right)=\tau\left(\left.\beta\right|_{o(k)}\right)$ for some $k$. Then $K_{\gamma} \cap\left[f^{n}(x) ; f^{n}(y)\right]=\emptyset$ for any $\tau\left(\left.\alpha\right|_{o(k-1)}\right)<n \leq \tau\left(\left.\alpha\right|_{o(k)}\right)$ if and only if one of the following alternatives occurs. 
(i) $\alpha_{o(k)}=0=\beta_{o(k)}$.

(ii) $\alpha_{o(k)} \neq 0, \beta_{o(k)}=0$ and $\sigma^{o(k)}(\gamma)<\sigma^{o(k)}(\alpha)$.

(iii) $\alpha_{o(k)} \neq 0 \neq \beta_{o(k)}$ and either

$$
\sigma^{o(k)}(\alpha)<\sigma^{o(k)}(\gamma) \text { and } \sigma^{o(k)}(\beta)<\sigma^{o(k)}(\gamma)
$$

or

$$
\sigma^{o(k)}(\gamma)<\sigma^{o(k)}(\alpha) \text { and } \sigma^{o(k)}(\gamma)<\sigma^{o(k)}(\beta) \text {. }
$$

Proof. First we assume $k=1$. It may be assumed that $f \in U(I)$. If $\tau\left(\alpha_{o(1)}\right)=0$, there is nothing to prove; so we will exclude this case. Then there are two possibilities for $\alpha$. If $\alpha_{o(1)}=0$, then $o(1)>1$, and so it makes sense to consider $\left.\gamma\right|_{o(1)-1}$, when $\left.\gamma\right|_{o(1)-1}=\left.\mathbf{1}\right|_{o(1)-1}$ by the definition of $o(1)$. Let $a$ be the left endpoint of $I$ and $b$ be the right endpoint of $K_{\left.1\right|_{o(1)-1}}$. Since $f(I) \subset[a, b]$ and $f\left(K_{\eta}\right) \cap K_{\mathbf{1}_{o(1)-1}}=\emptyset$ for any $\eta \in \mathbb{Z}^{o(1)-1} \backslash\left\{\left.\mathbf{0}\right|_{o(1)-1}\right\}$, we get $f^{n}(x)<K_{\gamma}$ for any $0<n \leq \tau\left(\left.\alpha\right|_{o(1)-1}\right)$. Since $\alpha_{o(1)}=0, f^{n}(x)<K_{\gamma}$ for any $0<n \leq \tau\left(\left.\alpha\right|_{o(1)}\right)$.

The second possibility is $\alpha_{o(1)} \neq 0$. As before, $f^{n}(x)<K_{\gamma}$ for any $0<n \leq \tau\left(\left.\alpha\right|_{o(1)-1}\right)$. Write $f^{\tau\left(\left.\alpha\right|_{o(1)-1}\right)}(x)=u$ and assume $o(1)>1$. Since $f^{j+t 2^{o(1)-1}}(u) \in K_{j}^{o(1)-1}$ for any $0 \leq j<2^{o(1)-1}$ and any $t \geq 0$, we get $f^{j+t 2^{o(1)-1}}(u)<K_{\gamma}$ for any $0 \leq j<2^{o(1)-1}(j \neq 1)$ and any $t \geq 0$. Recall that $u \in K_{\left.\mathbf{0}\right|_{o(1)-1}, \alpha_{o(1)}}$ and $f^{t 2^{o(1)-1}}(u) \in K_{\left.\mathbf{0}\right|_{o(1)-1}, t-\left|\alpha_{o(1)}\right|}$ for any $0<t \leq\left|\alpha_{o(1)}\right|$. Moreover, $\left(K_{\left.\mathbf{0}\right|_{o(1)-1},-1} \cup K_{\left.\mathbf{0}\right|_{o(1)-1}, 1}\right) \subset\left(K_{\left.\mathbf{0}\right|_{o(1)-1},-l} ; K_{\left.\mathbf{0}\right|_{o(1)-1}, l}\right)$ for any $l \geq 2$. Notice that $\left.\gamma\right|_{o(1)}=\left.\mathbf{1}\right|_{o(1)-1}, 0$, from which $f\left(K_{\left.\mathbf{0}\right|_{o(1)-1},-1}\right)=f\left(K_{\left.\mathbf{0}\right|_{o(1)-1}, 1}\right)=$ $K_{\left.\gamma\right|_{o(1)}}$. Then we get $f^{1+t 2^{o(1)-1}}(u)<K_{\gamma}$ for any $0 \leq t<\left|\alpha_{o(1)}\right|-1$. Finally let $r=1+\left(\left|\alpha_{o(1)}\right|-1\right) 2^{o(1)-1}$. Now $f^{r}(u) \in K_{\left.\gamma\right|_{o(1)}}$, more precisely $\left.\Pi\left(f^{r}(u)\right)\right|_{o(1)}=\left.\gamma\right|_{o(1)}$ and $\sigma^{o(1)}\left(\Pi\left(f^{r}(u)\right)\right)=\sigma^{o(1)}(\alpha)$. Then $f^{r}(u)<K_{\gamma}$ (resp. $K_{\gamma}<f^{r}(u)$ ) if and only if $\sigma^{o(1)}(\gamma)<\sigma^{o(1)}(\alpha)$ (resp. $\sigma^{o(1)}(\alpha)<$ $\left.\sigma^{o(1)}(\gamma)\right)$. In short, $f^{n}(x)<K_{\gamma}$ for any $0<n \leq \tau\left(\left.\alpha\right|_{o(1)}\right)$ except possibly for the case $n=\tau\left(\left.\alpha\right|_{o(1)}\right)-2^{o(1)-1}+1$, when $f^{n}(x)<K_{\gamma}\left(\right.$ resp. $\left.K_{\gamma}<f^{n}(x)\right)$ if and only if $\sigma^{o(1)}(\gamma)<\sigma^{o(1)}(\alpha)$ (resp. $\sigma^{o(1)}(\alpha)<\sigma^{o(1)}(\gamma)$ ). Recall that we were assuming $o(1)>1$. It is easy to check that the same result also holds in the case $o(1)=1$.

A similar reasoning for $\beta$ and $y$ completes the proof of the lemma for the case $k=1$. The general case follows from this one and Lemma 2 .

Proof OF Proposition 8. Observe that there are some nonnegative integers $k$ and $l$ such that $f^{k}\left(K_{\alpha}\right) \subset f^{k}\left(K_{\theta}\right), f^{l}\left(K_{\beta}\right) \subset f^{l}\left(K_{\vartheta}\right)$, and then it is not restrictive to assume that $\alpha=\theta, \beta=\vartheta$. Now the proposition follows easily from Lemmas 4 and 5 . 
It remains only to characterize explicitly $\sim_{e}$. This is a much easier task. $\gamma \sim_{e} \theta$ if and only $g\left(Z_{\gamma}\right)$ and $g\left(Z_{\theta}\right)$ are mapped to the same point by some iterate of the renormalization map $\nu$ (cf. the beginning of Section 2).

Proposition 9 Let $\gamma, \theta \in \mathcal{S}$. Then $\gamma \sim_{e} \theta$ if and only if there is a $k$ such that $\sigma^{k}(\gamma)=\sigma^{k}(\theta)$.

Proof. Let us prove the "only if" part. Suppose not. Then it may be assumed that there is a sequence $(i(n))_{n=1}^{\infty}$ such that $\gamma_{i(n)}=0, \theta_{i(n)}=1$ and $i(n+1)>i(n)+1$ for any $n$. Now choose $x, y \in K$ with $\alpha=\Pi(x)$, $\beta=\Pi(y)$ defined as follows. Put $\alpha_{i}=\beta_{i}=2$ for any $i \neq i(n), i \neq i(n)+1$, $n \geq 1$, and $\alpha_{i(n)}, \alpha_{i(n)+1}=2,4, \beta_{i(n)}, \beta_{i(n)+1}=6,2$ for any $n$. We have $\tau\left(\alpha_{\theta}^{j}\right)=\tau\left(\beta_{\theta}^{j}\right)$ for any $j$ (because $\left(\alpha_{\theta}^{j}\right)_{1}=4,\left(\beta_{\theta}^{j}\right)_{1}=2$ is impossible, and $\left(\alpha_{\theta}^{j}\right)_{o_{\theta}(j)-o_{\theta}(j-1)}=2,\left(\beta_{\theta}^{j}\right)_{o_{\theta}(j)-o_{\theta}(j-1)}=6$ is impossible as well $)$, and then from the comment below the statement of Proposition 8, $x \sim_{\theta}^{s} y$. On the other hand, $\tau\left(\left.\alpha\right|_{o_{\gamma}(k)}\right) \neq \tau\left(\left.\beta\right|_{o_{\gamma}(k)}\right)$ if $o_{\gamma}(k)=i(n)$ for some $n$; so $x \not_{\gamma}^{s} y$ by Proposition 8. This is a contradiction.

Let us prove now the "if" part. Suppose that $x \sim_{\gamma}^{s} y$ but $x \not_{\theta}^{s} y$ for some $x, y \in K$. Then there is a strictly increasing sequence $(n(j))_{j=1}^{\infty}$ such that $K_{\theta} \cap\left[f^{n(j)}(x) ; f^{n(j)}(y)\right] \neq \emptyset$ for any $j$. If $\tau\left(\left.\theta\right|_{k}\right) \leq \tau\left(\left.\gamma\right|_{k}\right)$, then $f^{l}\left(K_{\theta}\right)=K_{\gamma}$ for some $l$ and $K_{\gamma} \cap\left[f^{n(j)+l}(x) ; f^{n(j)+l}(y)\right] \neq \emptyset$ for any $j$, a contradiction. If $\tau\left(\left.\theta\right|_{k}\right)>\tau\left(\left.\gamma\right|_{k}\right)$, then there is an $m$ such that $f^{m}\left(K_{\left.\gamma\right|_{k}}\right)=K_{\left.\theta\right|_{k}}$ and $\left.f^{m}\right|_{K_{\left.\gamma\right|_{k}}}$ is monotone. By Lemma 3 and Proposition 6 , we can choose all $n(j)$ large enough so that $f^{n(j)-m}(x), f^{n(j)-m}(y) \in K_{\gamma \mid k}$ for any $j$. Since $f^{m}\left(K_{\gamma}\right)=K_{\theta}$, $K_{\gamma} \cap\left[f^{n(j)-m}(x) ; f^{n(j)-m}(y)\right] \neq \emptyset$ by Lemma 1 . This is impossible.

Remark 1 From Proposition 9 we easily get that $\gamma \sim_{e} \theta$ if and only if $\gamma \preceq \theta$ or $\theta \preceq \gamma$. (Recall that $\preceq$ was defined at the end of Section 2.) Then fix $\gamma \in \mathcal{S}$ and define $\mathcal{A}=\left\{\theta \in \mathbb{Z}^{\infty}: \theta \preceq \gamma\right\}$, which is countable and closed for $\preceq$. According to Proposition 4 , there is an $f \in W([0,1])$ such that, for any $\alpha \in \mathbb{Z}^{\infty}, K_{\alpha}$ is non-degenerate if and only if $\alpha \in \mathcal{A}$. For this function $f$, $x \sim^{s} y$ if and only if $x \sim_{\gamma}^{s} y$.

The following theorem summarizes our previous results and gives the desired description of all maximal scrambled sets of any $f \in W(I)$.

Theorem 1 Let $f \in W(I)$ and $S \subset I$. Then we have

(i) $f$ is chaotic if and only if it has some essential sequence.

(ii) Suppose $f$ is chaotic. Then $S$ is a maximal scrambled set of $f$ if and only if there is $a \sim^{i}$-class $E$ such that $S \subset E$ and $S$ contains exactly one representative from every one of the $\sim^{s}$-classes included in $E$. 
Moreover, the equivalence relations $\sim^{i}$ and $\sim^{s}$ can be explicitly described (respectively, Proposition 6 and Propositions 7, 8 and 9). In particular, every maximal scrambled set of $f$ is uncountable.

Notice that Proposition 7 is the only point where we make use of the specific features of $f$ (apart of course from $f \in W(I)$ ).

\section{Final Remarks and Open Questions}

Note that the set $\mathcal{E}$ from Proposition 7 can be infinite (countable), and in this case our characterization of $\sim^{s}$ turns out to be fairly complicated. Thus it would be interesting to find a minimal set $\mathcal{E}^{\prime} \subset \mathcal{S}$ (maybe not necessarily $\left.\mathcal{E}^{\prime} \subset \mathcal{E}\right)$ such that for any $x, y \in K, x \sim^{s} y$ if and only if $x \sim_{\gamma}^{s} y$ for every $\gamma \in \mathcal{E}^{\prime}$. If particular, in [9] we characterized the cases for which there is a $\gamma$ such that, for any $x, y \in K, x \sim^{s} y$ if and only if $x \sim_{\gamma}^{s} y$. (Then we could choose $\mathcal{E}^{\prime}=\{\gamma\}$.) However, Proposition 7 cannot be improved in general, even in the case when $\mathcal{E}$ is infinite. This is shown in the following example.

In order to shorten the notation, for any $\alpha \in \mathbb{Z}^{\infty}$ and $j \geq 1$, we will write $\underline{\alpha}^{j}=\left.\sigma^{(5+j / 2)(j-1)}(\alpha)\right|_{j}$ and $\bar{\alpha}^{j}=\left.\sigma^{5(j-1)+(j+1) j / 2}(\alpha)\right|_{5}$. Also, $\lambda^{n, m} \in\{0,1\}^{n}$, $1 \leq m \leq n$, will be defined by $\lambda_{n+1-m}^{n, m}=0$ and $\lambda_{i}^{n, m}=1$ if $i \neq n+1-m$. Now for any $n \geq 1$, define $\gamma[n] \in \mathcal{S}$ by $\underline{\gamma[n]^{j}}=\lambda^{j, j}$ if $j<n$ and $\underline{\gamma[n]^{j}}=\lambda^{j, n}$ if $j \geq n$, and $\overline{\gamma[n]}^{j}=\lambda^{5,3}$ for any $j$. Specifically,

$$
\begin{aligned}
\gamma[1] & =0 ; 1,1,0,1,1 ; 1,0 ; 1,1,0,1,1 ; 1,1,0 ; 1,1,0,1,1 ; 1,1,1,0 ; \ldots, \\
\gamma[2] & =0 ; 1,1,0,1,1 ; 0,1 ; 1,1,0,1,1 ; 1,0,1 ; 1,1,0,1,1 ; 1,1,0,1 ; \ldots, \\
\gamma[3] & =0 ; 1,1,0,1,1 ; 0,1 ; 1,1,0,1,1 ; 0,1,1 ; 1,1,0,1,1 ; 1,0,1,1 ; \ldots, \\
\gamma[4] & =0 ; 1,1,0,1,1 ; 0,1 ; 1,1,0,1,1 ; 0,1,1 ; 1,1,0,1,1 ; 0,1,1,1 ; \ldots, \\
& \ldots
\end{aligned}
$$

and so on. (Here the semicolons delimit the $\gamma[n]^{j}$ and $\overline{\gamma[n]}^{j}$ blocks.) The set $\mathcal{A}=\left\{\theta \in \mathbb{Z}^{\infty}: \theta \preceq \gamma[n]\right.$ for some $\left.n \geq 1\right\}$ is countable and closed for $\preceq$. So by Proposition 4 there is an $f \in W([0,1])$ with $\mathcal{E}=\{\gamma[n]: n \geq 1\}$. Now suppose that $\mathcal{E}^{\prime} \subset \mathcal{S}$ is such that, for any $x, y, x \sim^{s} y$ if and only if $x \sim_{\gamma}^{s} y$ for any $\gamma \in \mathcal{E}^{\prime}$. We will prove that there is a bijection $\psi: \mathcal{E} \rightarrow \mathcal{E}^{\prime}$ with $\gamma[n] \sim_{e} \psi(\gamma[n])$ for any $n$. In many stages of the following argument, we will have to check $x \sim_{\theta}^{s} y$ for several $x, y \in K$ and $\theta \in \mathcal{S}$. In each case this is a routine application of Proposition 8, so we will not go into the details.

First of all we prove that if $\gamma$ is such that $x \sim_{\gamma}^{s} y$ for any $x, y$ with $x \sim^{s} y$, then there is an $l$ such that $\gamma \sim_{e} \gamma[l]$. We will accomplish it through three successive steps.

Claim 1. There is a $k$ such that $\bar{\gamma}^{j}=\lambda^{5,3}$ for any $j>k$. 
First of all we show that there is an $m$ for which $\bar{\gamma}_{i}^{j}=1$ for any $i \neq 3$ and $j>m$. Otherwise we could find a strictly increasing sequence $(j(n))_{n=1}^{\infty}$ and an $r \in\{1,2,4,5\}$ such that $\bar{\gamma}_{r}^{j(n)}=0$ for any $n$. Let us say that $\bar{\gamma}_{r}^{j(n)}$ is the $i(n)$ th term of the sequence $\gamma$ for any $n$. Then choose $x, y \in K$ with the sequences $\alpha=\Pi(x), \beta=\Pi(y)$ defined by $\alpha_{i(n)}, \alpha_{i(n)+1}=1,2, \beta_{i(n)}, \alpha_{i(n)+1}=3,1$ for any $n$ and $\alpha_{i}=\beta_{i}=1$ otherwise. We have $x \sim^{s} y$ but $x \chi_{\gamma}^{s} y$, a contradiction.

Now assume that Claim 1 does not hold. According to the paragraph above, there must exist a strictly increasing sequence $(k(n))_{n=1}^{\infty}$ such that $\bar{\gamma}^{k(n)}=\left.\mathbf{1}\right|_{5}$ for any $n$. Then take some $x, y$ such that $\alpha=\Pi(x), \beta=\Pi(y)$ are defined by $\bar{\alpha}^{j}=3,0,1,-1,1, \bar{\beta}^{j}=1,1,1,-1,1$ for any $j$ and $\alpha_{i}=\beta_{i}=1$ otherwise. Again $x \sim^{s} y$ but $x \chi_{\gamma}^{s} y$, a contradiction.

Claim 2. There is a $k$ such that $\underline{\gamma}^{j} \in\left\{\lambda^{j, m}: 1 \leq m \leq j\right\}$ for any $j>k$.

Suppose not. Two possibilities appear. The first one is that there is a strictly increasing sequence $(j(n))_{n=1}^{\infty}$ such that $\gamma^{j(n)}=\left.\mathbf{1}\right|_{j(n)}$ for any $n$. Then take some $x, y$ for which the corresponding $\alpha, \beta$ are defined by $\bar{\alpha}^{j}=-1,1,1,3,0, \bar{\beta}^{j}=-1,1,1,1,1$ for any $j$ and $\alpha_{i}=\beta_{i}=1$ otherwise. Again we get $x \sim^{s} y$, but $x \chi_{\gamma}^{s} y$.

The second possibility is that there is a strictly increasing sequence $(k(n))_{n=1}^{\infty}$ such that for any $n \underline{\gamma}^{k(n)}$ has at least two terms equal to zero. For each $n$, let $i(n)$ be the last index with $\underline{q}_{i(n)}^{k(n)}=0$. It is not restrictive to assume that either $i(n)=k(n)$ for any $n$ or $i(n)<k(n)$ for any $n$. In the first case put $\underline{\alpha}^{k(n)}=\beta^{k(n)}=\gamma^{k(n)}$ and $\bar{\alpha}_{1}^{k(n)}=2, \bar{\beta}_{1}^{k(n)}=-2$ for any $n$ and define $\alpha_{i}=\beta_{i}=1$ otherwise. In the second case for any $n$ similarly define $\underline{\alpha}_{i}^{k(n)}=\underline{\beta}_{i}^{k(n)}=\underline{\gamma}_{i}^{k(n)}$ if $1 \leq i \leq i(n)$ and $\underline{\alpha}_{i(n)+1}^{k(n)}=2, \underline{\beta}_{i(n)+1}^{k(n)}=-2$, and put $\alpha_{i}=\beta_{i}=1$ otherwise. In each case we get $x \sim^{s} y$ while $x \chi_{\gamma}^{s} y$.

Claim 3. There are some $k$ and $l$ such that $\underline{\gamma}^{j}=\lambda^{j, l}$ for any $j>k$.

Again assume the contrary. Since Claim 2 holds, it is clearly possible to choose a sequence $(j(n))_{n=1}^{\infty}$ such that $j(n+1)-j(n)>1$ and $\underline{\underline{\gamma}}^{j(n)}=\lambda^{j(n), r(n)}$, $\underline{\gamma}^{j(n)+1}=\lambda^{j(n)+1, s(n)}$, with $r(n)<s(n)$ for any $n$. Now define $\underline{\alpha}^{j(n)}=$ $\underline{\beta}^{j(n)}=\underline{\gamma}^{j(n)}, \bar{\alpha}^{j(n)}=\bar{\beta}^{j(n)}=\lambda^{5,3}$ and $\underline{\alpha}_{j(n)+2-s(n)}^{j(n)+1}, \underline{\alpha}_{j(n)+3-s(n)}^{j(n)+1}=0,2$, $\underline{\beta}_{j(n)+2-s(n)}^{j(n)+1}, \underline{\beta}_{j(n)+3-s(n)}^{j(n)+1}=0,-2$ for any $n$, and put $\alpha_{i}=\beta_{i}=1$ otherwise. Since $s(n) \geq 2$ for any $n, \alpha$ and $\beta$ are well defined. Also, $x \sim^{s} y$ while $x \not_{\gamma}^{s} y$.

From Claims 1,2 and 3 we get as desired that if $\gamma$ is such that $x \sim^{s} y$ implies $x \sim_{\gamma}^{s} y$ for any $x, y \in K$, then there is an $l$ such that $\gamma \sim_{e} \gamma[l]$. To finish, fix $r \geq 1$ and choose $x, y$ in such a way that their corresponding $\alpha, \beta$ satisfy $\underline{\alpha}_{j+1-r}^{j}, \underline{\alpha}_{j+2-r}^{j}=0,2, \underline{\beta}_{j+1-r}^{j}, \underline{\beta}_{j+2-r}^{j}=0,-2$ for any $j>r$ and $\alpha_{i}=\beta_{i}=1$ otherwise. Then $x \sim_{\gamma[n]}^{s} y$ for any $n \neq r$ but $x \mathcal{\psi}_{\gamma[r]}^{s} y$. 
On the other hand, it would also be interesting to get a clearer information on the size of every $\sim^{s}$-class, in particular when a $\sim^{s}$-class $F$ is minimal; that is, the cases when $x, y \in F$ if and only if $\theta=\mathrm{n}(\Pi(x)), \vartheta=\mathrm{n}(\Pi(y))$ imply $\sigma^{k}(\theta)=\sigma^{k}(\vartheta)$ for some $k$. We have solved this problem in [9] for the case $\mathcal{E}=\{\gamma\}$.

Finally, it seems plausible that a similar approach to that of this paper could be useful for describing all maximal scrambled sets of general (not necessarily unimodal) functions of type $2^{\infty}$, the point being that these functions verify a "kind" of Proposition 1. (See Theorem 3.5 from Smítal [17].) For example, an analogous version of Proposition 6 is proved in Balibrea Gallego and Jiménez López [1].

\section{References}

[1] F. Balibrea Gallego and V. Jiménez López, A characterization of chaotic functions with entropy zero via their maximal scrambled sets, Math. Bohemica 120 (1995), 293-298.

[2] L. Block, Homoclinic points of mappings of the interval, Proc. Amer. Math. Soc., 72 (1978), 576-580.

[3] A. M. Bruckner and T. Hu, On scrambled sets and chaotic functions, Trans. Amer. Math. Soc., 301 (1987), 289-297.

[4] P. Collet and J. P. Eckmann, Iterated Maps on the Interval as Dynamical Systems. Progress in Physics 1, Birkhäuser, Boston, 1980.

[5] B. S. Du, Smooth weakly chaotic interval maps with zero topological entropy, in Proceedings of the International Conference on Dynamical Systems and Related Topics (Nagoya, Japan, 1990), pages 72-79, World Sci. Publishing, Singapore, 1991.

[6] J. Harrison, Wandering intervals, in Dynamical Systems and Turbulence, Lecture Notes in Mathematics, 898, pages 154-163, Springer, Berlin, 1981.

[7] V. Jiménez López, $C^{1}$ weakly chaotic functions with zero topological entropy and non-flat critical points, Acta Math. Univ. Comenian., 60(2) (1991), 195-209.

[8] V. Jiménez López, Large chaos in smooth functions of zero topological entropy, Bull. Austral. Math. Soc., 46 (1992), 271-285.

[9] V. Jiménez López, Maximal scrambled sets for simple chaotic functions, to appear in Pusl. mat. 
[10] T. Y. Li and J. A. Yorke, Period three implies chaos, Amer. Math. Monthly, 82 (1975), 985-992.

[11] M. Martens, W. C. de Melo and S. J. van Strien, Julia-Fatou-Sullivan theory for real one-dimensional dynamics, Acta Math., 168 (1992), 271-318.

[12] J. Milnor and W. Thurston, On iterated maps of the interval, in Dynamical Systems, pages 465-563, Springer, Berlin, 1988.

[13] M. Misiurewicz, Structure of mappings of an interval with zero entropy, Inst. Hautes Études Sci. Publ. Math., 53 (1981), 5-16.

[14] M. Misiurewicz, Chaos almost everywhere, in Iteration Theory and its Functional Equations, Lecture Notes in Mathematics, 1163, pages 125-130, Springer, Berlin, 1985.

[15] M. Misiurewicz and J. Smítal, Smooth chaotic functions with zero topological entropy, Ergod. Th. and Dynam. Sys., 8 (1988), 421-424.

[16] C. Preston, Iterates of Maps on an Interval, Lecture Notes in Mathematics, 999, Springer, Berlin, 1983.

[17] J. Smítal, Chaotic functions with zero topological entropy, Trans. Amer. Math. Soc., 297 (1986), 269-282.

[18] S. J. van Strien, Smooth dynamics on the interval, in New Directions in Dynamical Systems, (eds. T. Bedford and J. Swift), pages 57-119, Cambridge Univ. Press, 1988.

[19] A. N. Šarkovskii, On cycles and the structure of a continuous map, Ukrain. Mat. Ž., 17 (1965), 104-111, in Russian. 